\title{
Hierarchical Fault Diagnosis and Health Monitoring in Satellites Formation Flight
}

\author{
A. Barua, Student Member, IEEE, and K. Khorasani, Member, IEEE
}

\begin{abstract}
Current spacecraft health monitoring and fault diagnosis practices involve around-the-clock limit-checking and trend analysis on large amount of telemetry data. They do not scale well for future multi-platform space missions due the size of the telemetry data and an increasing need to make the long-duration missions cost-effective by limiting the operations team personnel. The need for efficient utilization of telemetry data achieved by employing machine learning and reasoning algorithms has been pointed out in the literature for enhancing diagnostic performance and assisting the lessexperienced personnel in performing monitoring and diagnosis tasks. In this paper, we develop a systematic and transparent fault diagnosis methodology within a hierarchical fault diagnosis framework for a satellites formation flight. We present our proposed hierarchical decomposition framework through a novel Bayesian Network (BN) whose structure is developed from the knowledge of component health state dependencies. We have developed a methodology for specifying the network parameters that utilizes both node fault diagnosis performance data and domain experts' beliefs. Our proposed model development procedure reduces the demand for expert's time in eliciting probabilities significantly. Our proposed approach provides the ground personnel with an ability to perform diagnostic reasoning across a number of subsystems and components coherently. Due to the unavailability of real formation flight data, we demonstrate the effectiveness of our proposed methodology by using synthetic data of a leader-follower formation flight architecture. Although our proposed approach is developed from the satellite fault diagnosis perspective, it is generic and is targeted towards other types of cooperative fleet vehicle diagnosis problems.
\end{abstract}

Index Terms-Fault Diagnosis; Model-based Reasoning; Bayesian Networks; Integrated Vehicle Health Management; Decision Support Systems; Spacecraft Formation Flight.

\section{INTRODUCTION}

$\mathbf{I}$ NTEGRATED Vehicle Health Management (IVHM) refers to the integration of techniques and technologies to provide a health management system for a vehicle or fleet of vehicles. Although the requirements are unique to a specific type of vehicle, health management has become increasingly important to automotive, commercial and military aircraft, rotorcraft, unmanned and manned vehicles, spacecraft, and satellites. In the space and commercial aviation sectors, the concept of IVHM is used to describe the automation of activities that are performed onboard as well as offboard by the ground support teams and maintenance personnel. Fault diagnosis is the part of an IVHM

Manuscript received dd/mm/yyyy, This research is supported in part by a Strategic Projects Grant and a Discovery Grant from the Natural Sciences and Engineering Research Council of Canada (NSERC).

The authors are with Department of Electrical \& Computer Engineering, Concordia University, 1455 De Maisonneuve Blvd. West, Montreal, Canada H3G 1M8. Corresponding author: K. Khorasani (E-mail: kash@ece.concordia.ca) system which aims to identify the root causes of faults and performance degradations.

In the case of satellites that operate in near-Earth orbits, it has been possible to manage and operate these systems through additional design margins and extensive ground-based monitoring and control efforts. Fault diagnosis and health monitoring in the Earth orbiting single spacecraft missions are mostly accomplished by human operators at ground through around-the-clock limitchecking and trend analysis on large amount of telemetry data by utilizing software tools. Current spacecraft diagnosis practices do not scale well for future multi-platform space missions due to the size of the telemetry data and an increasing need to make the long-duration missions cost-effective by limiting the operations team personnel. On the other hand, the effectiveness of spacecraft autonomy, which may be an ideal solution to this problem, is yet to be fully demonstrated. This is mainly constrained due to the existence of perceived risks for a fully autonomous system, which has necessitated that the expert human operators be involved in the spacecraft operations and diagnosis processes [1]. Furthermore, in order to enhance the diagnostic performance and assist the lessexperienced personnel in performing monitoring and diagnosis tasks at ground stations, there is a need for efficient utilization of the telemetry data [2].

Within an IVHM framework, offboard diagnosis of various components and subsystems are carried out by employing different types of reasoning algorithms. It is well-known within the Artificial Intelligence (AI) community that the diagnostic reasoning methods [3] are commonly classified into three main categories, namely (1) Case Based Reasoning (CBR), (2) Rule Based Reasoning (RBR), and (3) Model Based Reasoning (MBR). Other approaches are mainly pure data-driven that include machinelearning (ML) and Neural Network (NN)-based strategies. The MBR approach includes graph-based models such as logical causal graph models as well as Bayesian network (BN) models. The fault diagnosis approach developed in this paper is basically a model-based reasoning method that utilizes a Bayesian network model which we have identified as the Component Dependency Model (CDM) in the subsequent paragraphs and sections. As an alternative to the above-mentioned AI-based methods, model based Fault Detection and Isolation (FDI) methods [4] are extensively developed and utilized by the control community. Model based FDI methods are primarily based on precise mathematical models of the system under consideration. In our work, we use the terms "fault diagnosis", "fault identification" and "fault isolation" interchangeably.

For complex systems such as satellites, it is often the case that different design and development teams are involved in developing diagnostic algorithms for various components and subsystems. When these algorithms are employed independently and in isolation for diagnosing a specific component or subsystem, correlating faults that are identified at separate locations will lead 
to difficulties in assessing the overall system health. Therefore, a decision support system should be developed that would provide the ground personnel with an ability to perform diagnostic reasoning coherently. The focus of this work is to develop a methodology for ground station based diagnosis of complex multi-vehicle systems such as a formation flight of satellites where telemetry data is available, and access to precise mathematical models of the system under consideration is limited. Since it is desired that the diagnosis model would provide decision support to human experts, it is reasonable to decompose the overall system into simpler subcomponents, and to develop a Bayesian network-based (whose capability for human-like reasoning under uncertainties are well-known) fault diagnosis model to relate the faults that are occurring at the subcomponents. This is the rationale behind developing a Bayesian network type model in the present work. Our proposed Bayesian network-based diagnosis model is generic in the sense that it does not impose any restrictions on the type of diagnosis algorithms that one may employ at a given node of the model as long as its performance evaluation matrix (this will be discussed later in detail) is available.

To address the above problems and requirements for coherent diagnostic reasoning, in this paper we develop a systematic and transparent fault diagnosis methodology within the hierarchical fault diagnosis concepts and framework that we introduced in [5], [6] for multi-platform space systems or satellites formation flight. In this framework, as shown in Figure 1(a), we consider the satellites in a formation flight system as a set of hierarchically decomposed modules where entities at different levels correspond to formation, system, subsystem, etc. depending on the location of the entity in the hierarchy.

We present our proposed hierarchical decomposition by a Component Dependency Model (CDM) by using a novel Bayesian network (BN) [7], [8] structure. The structure of our CDM is determined from the knowledge of the component health state dependencies. A methodology is developed for specifying the CDM parameters that quantify the health state dependencies and domain experts' beliefs. The present work is an extension and a complete version of our earlier work [9], [10] on spacecraft fault diagnosis. Although, our proposed approach is developed from a satellite fault diagnosis perspective, it is generic and can be targeted towards other types of cooperative fleet vehicle diagnosis as long as it is viable to accommodate the unique requirements of the application area.

\section{A. Related Work}

A hierarchical Fault Detection, Isolation and Recovery (FDIR) concept for a single satellite system was presented in [11] in order to streamline and manage FDIR designs of multiple teams across a large number of spacecraft subsystems during various project phases. However, the authors in [11] present the concept mainly from the FDIR task management perspective without providing details on the systematic development of frameworks and methodologies. A generic hierarchical fault diagnosis approach is available in [3]; however, the properties of their hierarchical structure are much restrictive for applications to complex systems with multiple interactive subsystems. Furthermore, the structure does not take into account uncertainty that is related to component interactions or the health state dependencies. The applicability of hierarchical fault diagnosis methodologies that are based on the discrete-event systems theory is available in [12]. The method in
[12] is restricted to systems that are characterized by discrete events, and does not take into account uncertainties in the diagnosis model. A systems analysis method known as the fault propagation analysis (FPA) is available in [13] that facilitates a systematic design of fault tolerant control systems by identifying all possible faults in various components and their effects on the system. The method requires an in-depth knowledge of the components interactions and serves mostly as an analysis tool for early design modifications as opposed to a tool for operational fault diagnosis which is the primary focus of our current work. Furthermore, unlike our proposed method, FPA does not address uncertainties in the fault manifestations at different components of the FPA model.

Rule-based reasoning have been extensively used in various applications [14]-[20] including fault diagnosis. The author in [20] has pointed out that when the system or the application domain is very large and complex, an entirely rule-based representation and associated inference leads to a large and inefficient knowledge base, causing a poor quality in diagnosis. The author in [20] has reported a method of data analysis that is intended for autonomous real-time fault detection and characterization in spacecraft by utilizing both rule-bases and causal system models without providing much detail on how the models are developed and integrated. The rule-based diagnosis schemes in [17]-[19] appear to be suitable for fault diagnosis of a small set of actuators that are operated and diagnosed in isolation. Eventhough a number of research on dynamic neural networks [21]-[25] and machine learning techniques [22], [26] have been related to the fault diagnosis of subsystem components, they do not provide details on how the methodologies can be applied in the presence of a large number of interactive components and subsystems. Furthermore, on-board design and implementation of model-based FDI methods that include parameter estimation, robust observers and filter design techniques [4], [27]-[29] may be cost-prohibitive for a large number of subsystems and their components even if sufficiently accurate mathematical models are available.

An overview of the methods that are commonly employed for probability elicitation exhaustively from domain experts is available in [30]. The main drawback of these methods is their biased assessments by experts although various methods for interviewing experts have been developed in literature to reduce such biases. These include probability-scale method, gamblelike method, and the probability wheel method. Other methods are available in [31] and [32]. The methodology that we have developed in this work for quantifying our CDM parameters is the result of and is being motivated by the inapplicability of the existing methods (for example, the ones in [31], [32]) to our system. The method available in [31] utilizes domain-dependent constraints that are not relevant to our problem. The method that is available in [32] is also not applicable because it was developed for ranked nodes whose states are expressed on an ordinal scale which is mapped to a continuous, monotonically ordered, bounded numerical scale. Note that several belief or evidence propagation methods in BN are available in the literature [7], [8], and the methods require that the $\mathrm{BN}$ parameters of the nodes be specified numerically. Our focus in the present paper is on the BN-based fault diagnosis model development (structure and parameters) as opposed to the development of a belief propagation method.

It should be pointed out that the need for spacecraft autonomy is extensively discussed, and onboard planning, execution, diagnosis 
and recovery have been demonstrated in a deep space mission [33], [34]. A framework that facilitates reducing the operational cost at the ground station for NASA's Deep Space Network is available in [35]. However, in the operation of the Earth-orbiting satellites the implementation of such onboard autonomous diagnosis and recovery functions have yet to become a common practice due to cost considerations and perceived risks.

The organization of the remaining parts of this paper is as follows: in Section II, we develop a generic BN-based CDM that represents our proposed hierarchical framework for formation flight fault diagnosis. We discuss the purpose of our model, explain how the health states are defined at different nodes, develop procedure for specifying model parameters, and briefly discuss how evidences are generated at different nodes. In Section III, we provide a description of the formation flight system simulation that we have utilized for synthetic data generation, and demonstrate our proposed fault diagnosis approach. In Section IV, we present performance evaluation results, and discuss advantages, limitations and possible validation procedures of our model. Finally, conclusions are stated in Section V.

\section{BAyesian Network Model for Hierarchical FAult DIAGNOSIS}

Though the development of our proposed methodology is based on the health management of satellites formation flight, the methodology is generic enough to be applicable to other systems or a fleet of systems that require health monitoring decision support systems (DSS). Our proposed fault diagnosis strategy aims to perform diagnostic reasoning in complex systems such as a "formation flight system" by decomposing its complex structure hierarchically into simpler modules or components. This idea was introduced in our earlier work [5], [6]. The decomposition is driven by the need, from project management perspective, for supporting the development of the components/subsystems of the overall system by a number of teams and performing integration at the end. As illustrated by the perspective of the attitude and orbital control of the satellites in a formation flight in Figure 1(a), fault diagnosis at level 1 (subsystem component level) corresponds to sensor and actuator fault diagnosis. Fault diagnosis at level 2 (subsystem level) corresponds to different subsystems such as attitude, orbital, power, etc. Level 3 (system level) diagnosis corresponds to individual satellites. In level 4 (formation level) diagnosis, individual spacecraft in the formation are considered as different "components" of the formation flying system.

First, we take into consideration that even if a fault is originated in a subsystem component, the fault is assumed to have various levels of manifestations in the hierarchy. In other words, for performing diagnosis at different levels, it is assumed that fault symptoms/manifestations are available. As an example, consider the specific fault of "increase in friction" in the pitch axis reaction wheel (subsystem component). At the subsystem component level, one of the fault manifestations would be "high current drawn by the wheel motor". In the attitude control subsystem (ACS) level, one of its manifestations would be "deviation from the expected pitch angle". The system level manifestations can be described in terms of deviations from some relative subsystem specifications. Finally, at the formation level the fault manifestations can be "deviation from the expected relative attitude with respect to other satellites". Therefore, in this case, there is a need for identifying faults differently at various levels in the hierarchy based on the "level of abstraction" at a particular level and the manifestation of fault at that particular level. On the other hand, in the case of a system level anomaly that leads to a situation in which a wrong command is sent to the actuator, the subsystem component, or even the subsystem, it would follow the "wrong" command without being aware of the anomaly. Based on the above observations, the definition of an "level $l$ fault" [6] is formally stated as follows:

Definition 2.1 (Level l Fault [6]): A fault occurring in a system that is hierarchically decomposed into L levels is said to be an "level l fault" $(l=1,2, \ldots, L)$ and is denoted by fault $f_{k}^{l}(k$-th fault mode) if and only if its manifestations are only observable in the fault signatures that belong to level $l$ and in higher levels for the fault severity level(s) under consideration.

Distinguishing faults at different levels based on the above definition would allow one to avoid cycles in our Bayesian network (BN)-based fault diagnosis model which we will describe in the subsequent sections.

\section{A. Proposed Bayesian Network Model Structure and Node States}

We represent our proposed hierarchical decomposition with a novel Bayesian network-based Component Dependency Model (CDM) as shown in Figure 1(b). The entire system under consideration is described by a single node at the highest level and which consists of sub-components that are located at lower levels. We denote the $p$-th component at level $l$ in the hierarchy as $C_{p}^{l}$. For example, if we consider a 4-level decomposition of a fleet of systems as shown in Figure 1(a), for $l=1, C_{p}^{1}$ would correspond to the $p$-th sensor or actuator (subsystem component) whereas for $l=4, C_{1}^{4}$ would correspond to the "fleet". For the intermediate levels, i.e., $l=2$ and $l=3$, a component $C_{p}^{l}$ would correspond to the $p$-th subsystem and system, respectively. Let $L$ denote the total number of levels in the hierarchy, and for any $C_{p}^{l}$, the set of components that are parents of $C_{p}^{l}$ (as represented in Figure 1(b)) is denoted by $p a\left(C_{p}^{l}\right)$. At this point, it is important to describe the main objective of our proposed hierarchical fault diagnosis and health monitoring approach in detail. We intend to utilize our scheme as follows: when the faulty or healthy state of a node is observed by executing a diagnosis algorithm, the evidence (refer to Section II-C for the specific approach adopted in this paper) is introduced to our proposed CDM by instantiating that node. The evidence is then propagated in the CDM by utilizing a standard propagation algorithm (such as the junction tree algorithm, recursive conditioning algorithm, etc.). In the nodes that have updated health states corresponding to the faulty states with high probabilities, diagnosis algorithms are executed to confirm the hypotheses. When a fault evidence is determined at some intermediate level in the hierarchy, the evidence is propagated downwards to identify the component in which the fault has originated from. On the other hand, the evidence is propagated upwards to identify components that are probably affected by the fault, and to determine if higher level specifications are still possible to be accomplished since the diagnosis algorithms at higher levels are usually based on certain rules that check the system (or, system of system) level specifications. It is possible to encounter situations where there is no identification of a faulty state at a higher level, whereas a low level fault is actually identified at a lower level. However, in such cases, it is worthwhile to propagate the evidence upwards in the hierarchy to 


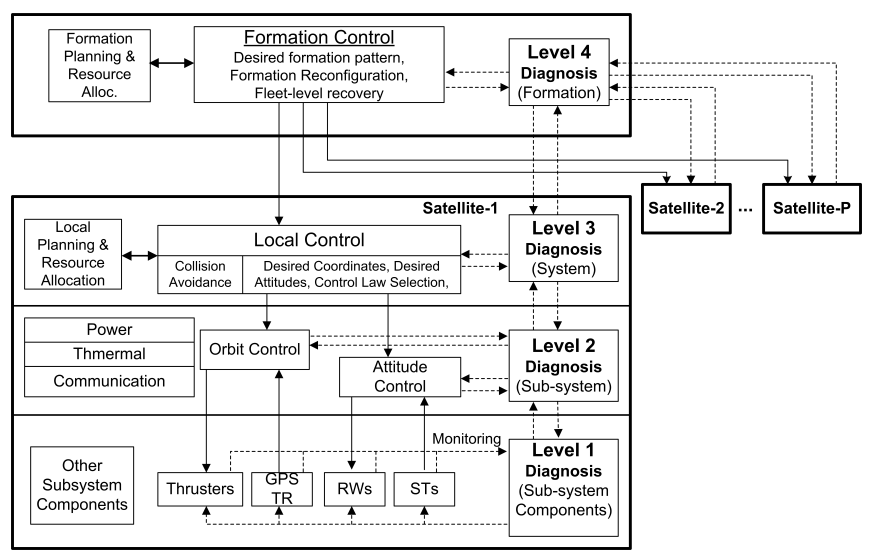

(a) A 4-level hierarchical fault diagnosis framework for a leader-follower satellites formation flight [6].

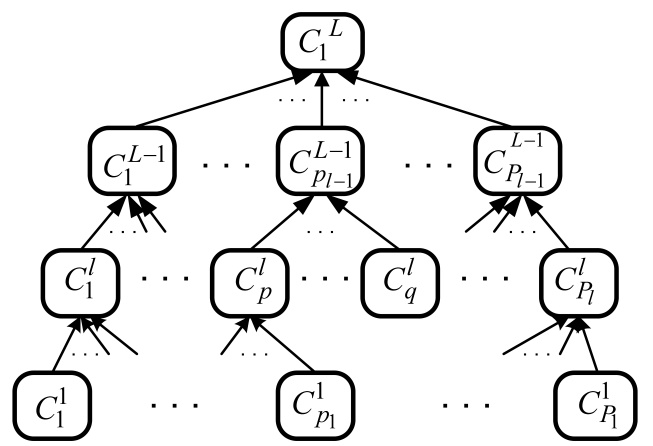

(b) Bayesian network representation of an $L$ level hierarchical decomposition.

Fig. 1. Proposed hierarchical decomposition of satellites formation flight.

identify the high level components that are possibly impacted by the identified fault. On the other hand, when the diagnosis at a higher level is accurate, it is worthwhile to propagate the evidence downwards in the hierarchy to identify the components where one should expect to observe fault manifestations eventhough fault identification cannot be performed at the current instant.

It should be noted that there are certain cost that is associated with performing fault diagnosis at each node in terms of data processing, algorithm development, validation and performance evaluation. Furthermore, in case of having a large number of components, it is natural from the users' resource considerations that the number of nodes that are to be actively/ round the clock monitored is as few as possible. Consequently, it is possible that diagnosis algorithms are not employed at some of the intermediate nodes but it is desired that the nodes be included in the diagnosis model to determine which subsystem or system a faulty node at lower levels belongs to. Such representation allows a systematic fault cause identification. In subsequent paragraphs, we will investigate a general case of an $L$ level hierarchical decomposition.

Node Health States: The possible states of a given node in our proposed CDM represent the health states of the corresponding component. It should be clear, according to Definition 2.1 that the origin of a fault (level $l$ fault) is at one of the nodes $C_{p}^{l}$ (refer to Figure 1(b)) for which $p a\left(C_{p}^{l}\right)=\emptyset$. If $p a\left(C_{p}^{l}\right) \neq \emptyset$, the states of the parent nodes have impact on the states of $C_{p}^{l}$, and the fault may manifest at $C_{p}^{l}$ after originating from some other node at lower levels. Depending on whether a node $C_{p}^{l}$ has parent nodes or not, we assign its health state as follows: given a component $C_{p}^{l}$ and its parents $p a\left(C_{p}^{l}\right)=\left\{C_{1}^{l-1}, \ldots, C_{m}^{l-1}, \ldots, C_{M}^{l-1}\right\}$, the possible health states $X_{p}^{l}$ of $C_{p}^{l}$ are represented as $X_{p}^{l}=$ $\left\{x_{0}, \ldots, x_{m}, \ldots, x_{M}\right\}$; where $x_{0}$ corresponds to the state "healthy $C_{p}^{l, "}$ and $x_{m}$ corresponds to the state "component $C_{m}^{l-1}$ fault in $C_{p}^{l,}$. If $p a\left(C_{p}^{l}\right)=\emptyset$, the possible health states $X_{p}^{l}$ of $C_{p}^{l}$ are represented as $X_{p}^{l}=\left\{x_{0}, \ldots, x_{k}, \ldots, x_{K}\right\}$; where $x_{k}$ corresponds to the level $l$ fault $f_{k}^{l}$ that originates at $C_{p}^{l}$.

Note that it is possible to represent an anomaly in a node that corresponds to multiple simultaneous faults by a health state of the node. However, the diagnosis algorithm that is employed at that node must be capable of distinguishing among say, two single faults and their simultaneous occurrences. In such a case, from fault identification perspective, the anomaly involving multiple faults can be treated as a "single fault" while generating a node performance evaluation matrix such as a confusion matrix [36]. For sake of simplicity, in this paper we do not consider health states $x_{m} \in X_{p}^{l}$ (or $x_{k} \in X_{p}^{l}$ ) that correspond to multiple fault scenarios.

It is worthwhile to note that in Bayesian modeling, it is required that the possible node's states are exhaustive and mutually exclusive to ensure that the entire state space is under consideration and the node is in a single state at a given instant. These requirements are satisfied in a practical environment by taking into account all the possible, or at least the dominant, faults corresponding to that node that are determined through the wellknown Failure Mode Effect and Criticality Analysis (FMECA) procedures. As mentioned above, node states are observed by executing appropriate fault diagnosis algorithms at that node. Therefore, evidence should be introduced to the network nodes when the states are identified by the diagnosis algorithms without ambiguity. It is possible to design diagnosis algorithms that provide information on such ambiguity as we have demonstrated in our earlier work [6], [9]. In the present work, we have employed fuzzy Rule Based Reasoning (RBR) to identify the states of a node and to generate evidences that are introduced to the node which we discuss in detail in Section II-C. However, our proposed hierarchical approach is generic for accommodating any type of reasoning algorithm; i.e., at a particular node, Case Based Reasoning (CBR) or Model Based Reasoning (MBR) algorithms may be employed as well.

\section{B. Determination of Model Parameters}

Parameters of our proposed Bayesian network-based CDM are the conditional probabilities that are specified in the form of Conditional Probability Tables (CPT). It is well-known that the CPT that is specified at $C_{p}^{l}$ has a number of parameters (conditional probabilities) that are exponential in the number of parents $p a\left(C_{p}^{l}\right)$; i.e., one must specify $P\left(X_{p}^{l} \mid p a\left(X_{p}^{l}\right)\right)$ for each configuration of the parents. As mentioned in Section IA, an overview of the methods that are commonly employed for probability elicitation from domain experts is available in [30], and the limitations of eliciting probabilities exhaustively with domain experts are well-known. Detailed discussions on the benefits and drawbacks of these methods are available in [30], and reviewing them is therefore beyond the scope of this paper.

In our case, elicitation of CPTs from the domain expert opinions will be difficult because as the possible number of faults becomes large in the parent nodes of a given node, the number of 
parent configurations will become too specific for the expert to specify a distribution of the node's health state. Furthermore, it is not reasonable to assume that real data corresponding to different faults and all of their combinations are available. Generating synthetic data for combinations of fault occurrences will be cost prohibitive and challenging, if not impossible, eventhough a high fidelity simulator is available. Therefore, a requirement for parameter learning from data is likely to impose a significant barrier in model development and deployment.

Note that existing methods for generating CPTs are not useful in our case because of the following reasons, namely, though noisy-OR [8] is a well established method, it applies only to boolean nodes. The method available in [31] utilizes domaindependent constraints that are not relevant to our problem. The method that is available in [32] is also not applicable because it was developed for ranked nodes whose states are expressed on an ordinal scale which is mapped to a continuous, monotonically ordered, bounded numerical scale. The above-mentioned difficulties in eliciting probabilities from experts and the unavailability of sufficient data that are always barriers in deploying diagnostic schemes for real systems, have motivated us to investigate alternative methods for generating CPTs.

Uncertainty Information: Recall that our central problem at hand is to manage and utilize the health observations that are available from different subsystems and components. A framework and methodology is proposed in our work for health monitoring of complex systems where it is desired that diagnostic decisions are to be made by taking into account the uncertainty that is associated with health state observations at different nodes in the hierarchy. The uncertainty is quantified by the conditional probabilities that, as indicated above, are commonly elicited by utilizing information from the various sources [37] such as: (a) physical or numerical models $(b)$ results of experiments or passive observations, and $(c)$ opinions of domain experts.

In the area of health monitoring, the performance metrics for fault detection and identification/isolation are specified separately for both temporal and static performance evaluations [38], [39]. Since our work is concerned with fault identification by using a probabilistic reasoning model, we concentrate on confusion matrices that are used to evaluate static performance of isolation algorithms, and which also provide statistical or probabilistic information.

In our proposed CDM, the health state of a given node is observed by first employing the most appropriate fault diagnosis algorithms that are feasible. Despite the fact that these algorithms are developed by different teams separately and are often proprietary to the teams, it is expected that the diagnosis algorithms that are employed at different nodes have their respective performance evaluation data available, in the form of confusion matrices [36]. A confusion matrix consists of the elements representing the proportion of correct and incorrect classification rates. It is possible to obtain the following conditional probabilities form the confusion matrix that is associated with a given node: $P\left(X_{p}^{l}=\right.$ $\left.x_{k} \mid I_{p}^{l}=x_{n}\right)$; where $N$ is the maximum possible value of $k$ (and $n$ ), $k=0, \ldots, N, n=0, \ldots, N$, and $I_{p}^{l}$ is the health state identification at the node. By utilizing these local conditional probabilities, one can derive the conditional probabilities that are necessary to specify $P\left(X_{p}^{l} \mid p a\left(X_{p}^{l}\right)\right)$, and qualify the uncertainty in our proposed CDM as described in the subsequent paragraphs.
Overview of the Proposed Procedure: In [40], it is argued that the care with which any given probability distribution needs to be elicited in a BN model depends strongly on the structure of the model and the queries that are intended to be processed. Our proposed procedure for CPT generation focuses on a set of initial distributions that are easily verifiable by a human expert. The idea is to construct a set of initial distributions from the information that is available in the confusion matrices, and provide a flexibility to a human expert for modifications, if necessary. There may not be a need for any modification if the expert agrees with the initial distributions. Therefore, instead of asking an expert to provide a new distribution, a procedure is developed to construct distributions that the expert can modify, if necessary, according to his/her belief. These initial distributions mainly correspond to non-simultaneous sub-component (parent node) faults within a component (child node). Another rational for providing the non-simultaneous sub-component faults significance is due to the fact that most diagnosis algorithms are designed by incorporating this assumption. Furthermore, it is reasonable to assume that the probability of occurring simultaneous $n$ component faults in a set of components decreases as the number of faults $n$ increases. Once the initial distributions are determined, remaining distributions are derived from these initial ones.

Initial Distributions: Consider a generic segment of our proposed model as shown in Figure 2, where the child node $C_{p}^{l}$ at level $l$ has $N$ parent nodes at level $l-1$; i.e., $p a\left(C_{p}^{l}\right)=$ $\left\{C_{1}^{l-1}, \ldots, C_{n}^{l-1}, \ldots, C_{N}^{l-1}\right\}$ and their corresponding number of health states are $\left(m_{1}+1\right), \ldots,\left(m_{n}+1\right), \ldots,\left(m_{N}+1\right)$. Consequently, the possible health sates of $C_{p}^{l}$ are $X_{p}^{l}=\left\{x_{0}, \ldots, x_{n}, \ldots, x_{N}\right\}$ and the possible health sates of the $n$-th parent node are $X_{n}^{l-1}=\left\{x_{i}\right\}$; $i=0,1, \ldots, m_{n}$. In other words, the possible number of parent configurations is $\prod_{n=1}^{N}\left(m_{n}+1\right)$. Our objective is to determine a CPT that specifies $P\left(X_{p}^{l} \mid X_{1}^{l-1}, \ldots, X_{N}^{l-1}\right)$. Let $I_{p}^{l}$ denotes the

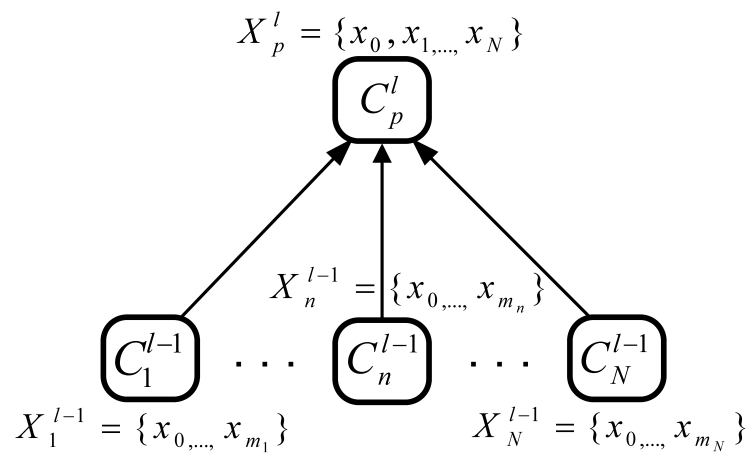

Fig. 2. Health states of a child node at level $l$ and its parent nodes at level $l-1$.

output of the health state identification algorithm that is employed at the node $C_{p}^{l}$. Hence, the possible outputs of $I_{p}^{l}$ correspond to the possible node health states $X_{p}^{l}$; i.e., $I_{p}^{l}=\left\{x_{0}, \ldots, x_{n}, \ldots, x_{N}\right\}$. From the confusion matrix it is possible to obtain the following information: $P\left(X_{p}^{l}=x_{k} \mid I_{p}^{l}=x_{n}\right)$; where, $k=0, \ldots, N$, and $n=0, \ldots, N$. Similarly, at level $l-1$, the information available at the $n$-th sub-component of $C_{p}^{l}$ are: $P\left(X_{n}^{l-1}=x_{k} \mid I_{p}^{l-1}=x_{i}\right)$; where $k=0,1, \ldots, m_{n}$ and $i=0,1, \ldots, m_{n}$.

In order to determine the $\prod_{n=1}^{N}\left(m_{n}+1\right)$ belief or probability distributions $P\left(X_{p}^{l} \mid X_{1}^{l-1}, \ldots, X_{N}^{l-1}\right)$ from the conditional probabilities above, first we focus on the distributions that correspond to 
single (non-simultaneous) component faults at level $l-1$ and the one that corresponds to the healthy states of all the components at level $l-1$. Our objective is to determine $N_{\lambda}+1$ initial distributions over $X_{p}^{l}$, where $N_{\lambda}=\sum_{j=1}^{N} m_{j}$. Note that these distributions correspond to the parent configurations which can be verified relatively easily by a human expert. The $N_{\lambda}$ initial distributions that correspond to the fault occurrences at level $l-1$ are in the following general form:

$$
\begin{array}{r}
P\left(X_{p}^{l} \mid X_{1}^{l-1}=x_{0}, \ldots, X_{n-1}^{l-1}=x_{0}, X_{n}^{l-1}=x_{i}, X_{n+1}^{l-1}=x_{0},\right. \\
\left.\ldots, X_{N}^{l-1}=x_{0}\right)
\end{array}
$$

where $n=1, \ldots, N$ and $i=1, \ldots, m_{n}$. The remaining one initial distribution is as follows:

$$
P\left(X_{p}^{l} \mid X_{1}^{l-1}=x_{0}, \ldots, X_{n}^{l-1}=x_{0}, \ldots, X_{N}^{l-1}=x_{0}\right)
$$

Computation of Initial Distributions: First, we note that there is a systematic pattern by which the health states at level $l-1$ are mapped to the health states $X_{p}^{l}$ at level $l$ in our proposed CDM which is as follows:

Observaton 2.1 (Health State Mapping): The health states $X_{p}^{l}$ of a component at level $l$ and its sub-components at level $l-1$ are mapped as follows:

- $X_{n}^{l-1}=x_{i} ; i=1, \ldots, m_{n}$ are mapped to the state $X_{p}^{l}=x_{n}$ for a given component at level $l-1$.

- $X_{n}^{l-1}=x_{0} ; n=1, \ldots, N$ (non-faulty states of multiple components) are mapped to a single state $X_{p}^{l}=x_{0}$.

It is important to note that according to the way node health states are mapped in our modes, and as stated in Observation 2.1, $X_{p}^{l}=x_{0}$ is to be considered "true" only when all the parent nodes are healthy; i.e., $X_{n}^{l-1}=x_{0} ; \forall n$. It is now reasonable to state the following assumption.

Assumption 2.1 (Independent Influences of Parent Nodes): Faults or faulty states of the components at level $l-1$ influence the component health states at level l independently.

Assumption 2.1 is particularly valid if the target severity range (refer to the Definition 2.1) is low and the components are monitored frequently enough so that the occurrences of faults in one component do not affect the fault identification in other components [6]. Based on this independence assumption, the distribution in (1) is approximated as follows

$$
\begin{gathered}
P\left(X_{p}^{l} \mid X_{1}^{l-1}=x_{0}, \ldots, X_{n-1}^{l-1}=x_{0}, X_{n}^{l-1}=x_{i}, X_{n+1}^{l-1}=x_{0},\right. \\
\left.\ldots, X_{N}^{l-1}=x_{0}\right) \\
\approx\left(P\left(X_{p}^{l}=x_{0} \mid X_{1, \ldots, N}^{l-1}=x\right), P\left(X_{p}^{l}=x_{1} \mid X_{n}^{l-1}=x_{i}\right),\right. \\
\ldots, P\left(X_{p}^{l}=x_{n-1} \mid X_{n}^{l-1}=x_{i}\right), P\left(X_{p}^{l}=x_{n} \mid X_{n}^{l-1}=x_{i}\right), \\
\left.P\left(X_{p}^{l}=x_{n+1} \mid X_{n}^{l-1}=x_{i}\right), \ldots, P\left(X_{p}^{l}=x_{N} \mid X_{n}^{l-1}=x_{i}\right)\right)
\end{gathered}
$$

where the first term is conditioned on the health states of all the parent sub-components at level $l-1$ with $x \neq x_{0}$ for the $n$-th sub-component, and $x=x_{0}$ otherwise. Since $P\left(X_{p}^{l}\right)=1$, the distribution in (3) is subjected to the constraint

$$
\sum_{j=1}^{N} P\left(X_{p}^{l}=x_{j} \mid X_{n}^{l-1}=x_{i}\right)=1
$$

As indicated above, the conditional probabilities that are available from the confusion matrices at levels $l$ and $l-1$ are local to the nodes at a given level. On the other hand, our problem is to quantify dependencies between levels $l$ and $l-1$. The difficulty is that due to different sensitivities of the diagnostic signals at the two levels there is no guarantee that whenever a fault is identified at level $l-1$ at a given instant, its manifestation at level $l-1$ is also identified at that instant as well or vice versa. One way to determine the dependencies is to conduct extensive experiments to observe the relative diagnostic performances of the nodes at the two levels for obtaining each CPT which is quite difficult, if not impossible. Alternatively, according to the way the health state mapping is set up in our model it is easy to see that whenever a faulty state $X_{n}^{l-1}=x_{i}$ is identified at level $l-1$, the component $C_{p}^{l}$ becomes faulty (since $C_{n}^{l-1}$ is a sub-component of $C_{p}^{l}$ ) whether the health state of $C_{p}^{l}$ is identified as $X_{p}^{l}=x_{n}$ or not. In the case where the fault is not identified al level $l$, the fault is latent in the sub-component $C_{n}^{l-1}$ within $C_{p}^{l}$. Based on the above observations, one can make approximations that if a state at level $l-1$ is faulty, the state at level $l$ is the corresponding faulty state as well. Consequently, one can utilize the conditional probabilities that are available from the confusion matrices at level $l-1$ to specify the distributions in (1) and (2). Therefore, to practically overcome an unrealistic requirement of conducting extensive experiments, we propose to quantify dependencies by introducing the notion of hierarchical health state agreement as follows:

\section{Definition 2.2 (Hierarchical Health State Agreement):}

Given the health state mapping in the Observation 2.1, and an identified fault that is manifested as $X_{p}^{l}=x_{n},(n \neq 0)$ at level $l$ and $X_{n}^{l-1}=x_{i},(i \neq 0)$ at level $l-1$, the health state identifications are in agreement if whenever $I_{p}^{l}=x_{n}$ at level $l$, $I_{n}^{l-1}=x_{i}$, at level $l-1$.

Based on the Definition 2.2, if $I_{p}^{l}$ and $I_{n}^{l-1}$ are known to be in agreement, given $I_{n}^{l-1}=x_{i}$, the probabilities of $X_{p}^{l}$ and $X_{n}^{l-1}$ are the same. However, it is necessary to specify the "degree of agreement" to quantify the Definition 2.2 in presence of the above-mentioned uncertainties. We propose the following policy to quantify the degree of agreement by a belief adjustment factor that is denoted by $h_{n, i}^{p, n}$ as follows:

$$
h_{n, i}^{p, n}= \begin{cases}a_{x_{i}}^{l-1} / a_{x_{n}}^{l} & \text { if } a_{x_{i}}^{l-1}<a_{x_{n}}^{l} \\ a_{x_{n}}^{l} / a_{x_{i}}^{l-1} & \text { if } a_{x_{i}}^{l-1}>a_{x_{n}}^{l}\end{cases}
$$

where $a_{x_{n}}^{l}$ is the accuracy with which the health state $x_{n}$ is identified at level $l$. The notion of accuracy is computed by constructing a "one-versus-all" decision matrix (as discussed in [6]) from the confusion matrix by following the procedure that is described next.

Let $\mathbf{C}_{\text {con }}$ denote an $(N+1) \times(N+1)$ confusion matrix that is associated with $N+1$ health states of a node at level $l$ in which the actual and the identified health states are along the rows and the columns, respectively. To compute the accuracy in identifying the $n$-th state, a $2 \times 2$ dimensional "one-versus-all" decision matrix $\mathbf{C}_{n}$ is constructed as follows. Let $c_{i, j}$ denote the element in the $i$-th row and the $j$-th column of $\mathbf{C}_{c o n}$, and $c_{i, j}^{\prime}$ denote the element in the $i$-th row and the $j$-th column of $\mathbf{C}_{n}$. The elements of the $\mathbf{C}_{n}$ matrix are computed from $c_{2,2}^{\prime}=c_{n, n}$, $c_{2,1}^{\prime}=\left(\sum_{k=1}^{N} c_{n, k}\right)-c_{n, n}, c_{1,2}^{\prime}=\left(\sum_{k=1}^{N} c_{k, n}\right)-c_{n, n}$, and $c_{1,1}^{\prime}=\left(\operatorname{sum}\left(\mathbf{C}_{\text {con }}\right)-c_{2,2}^{\prime}-c_{2,1}^{\prime}-c_{1,1}^{\prime}\right)$. The accuracy of identifying the $n$-th state is now defined as $a_{x_{n}}^{l}=\operatorname{trace}\left(\mathbf{C}_{n}\right) / \operatorname{sum}\left(\mathbf{C}_{n}\right)$. 
Similar procedure is followed to determine $a_{x_{i}}^{l-1}$. The superscripts $p, n$ in $h_{n, i}^{p, n}$ correspond to the $p$-th component $C_{p}^{l}$ and its $n$-th health state. The subscripts $n, i$ correspond to the $n$-th sub-component of $C_{p}^{l}$ and its $i$-th health state. It is important to note that the belief adjustment factor provides one's degree of belief (in terms of probabilities) about the health states that should be decreased when the level $l$ is changed. Therefore, if the probability of $X_{n}^{l-1}$ is known given certain condition $I$, to find the probability of $X_{p}^{l}$ given the same condition $I, P\left(X_{n}^{l-1} \mid I\right)$ should be multiplied by the belief adjustment factor. We consider $\left(1-h_{n, i}^{p, n}\right)$ to be a representative of the degree of disagreement.

It is not unusual that in most cases a diagnosis algorithm that is employed at a specific module or component meets the user specified accuracy (say, $\gamma_{s p e c}$ ) in identifying the component health states by using the test data. For example, in [36], the acceptance criteria for fault isolation/identification given a detection is recommended as $\gamma=0.95$ in a major component; i.e., the deployed fault identification algorithms should be capable of identifying $95 \%$ of the faults that are detected by the fault detection mechanism. Consequently, $\gamma_{s p e c} \leq a_{x_{n}}^{l} \leq 1, \gamma_{s p e c} \leq$ $a_{x_{i}}^{l-1} \leq 1$, and it follows that $0<h_{n, i}^{p, n} \leq 1$; where $h_{n, i}^{p, n}=1$ represents the highest degree of hierarchical agreement. Note that at any two consecutive levels it is possible to have low accuracies but high belief adjustment factors. Furthermore, it is important to point out that the above policy is not precise, since as stated in Observation 2.1 all the faulty health states of the $n$-th subcomponent at level $l-1$ are mapped to one health state $X_{p}^{l}=x_{n}$ at level $l$. Consequently, $a_{x_{n}}^{l}$ does not entirely correspond to the health state $X_{n}^{l-1}=x_{i},(i \neq 0)$. However, it should be clear that with the accuracy $\gamma_{s p e c} \leq a_{x_{i}}^{l-1} \leq 1$ in identifying $X_{n}^{l-1}=x_{i}$, $i=1, \ldots m_{n}$, the policy is expected to be well-behaved. Next, we determine the probability values that are corresponding to the approximated distribution in (3) which are categorized into the following three cases:

Case 1: Computation of $P\left(X_{p}^{l}=x_{k} \mid X_{n}^{l-1}=x_{i}\right)$ for $k=n$

This is the probability that the state $X_{p}^{l}$ is in its $n$-th faulty state given that the fault has been identified in its $n$-th subcomponent; i.e., $X_{n}^{l-1}=x_{i}$. Since all faulty states of a particular parent node are mapped to a single faulty state of the child node (refer to Observation 2.1), as long as the fault is identified at the $n$ th subcomponent $\left(X_{n}^{l-1} \neq x_{0}\right)$, it is desired that the state at level $l$ be $X_{p}^{l}=x_{n}$. Therefore, one must take into account both the correctly classified faults and the misclassified (with other faults in the subcomponent) faults at level $l-1$ while computing $P\left(X_{p}^{l}=x_{n} \mid X_{n}^{l-1}=x_{i}\right)$.

The probability $P\left(X_{p}^{l}\right)$ is conditioned on a faulty state which is identified by observing the output of $I_{n}^{l-1}$. Therefore, assuming a hierarchical health state agreement (Definition 2.2) with the belief adjustment factor $h_{n, i}^{p, n}$, we propose the following:

$$
\begin{aligned}
& P\left(X_{p}^{l}=x_{n} \mid X_{n}^{l-1}=x_{i}\right) \\
& \approx P\left(X_{p}^{l}=x_{n} \mid I_{n}^{l-1}=x_{i}\right) \\
& =h_{n, i}^{p, n}\left(P\left(X_{n}^{l-1}=x_{i} \mid I_{n}^{l-1}=x_{i}\right)\right. \\
& \left.\quad+\sum_{j \neq i, j=1}^{m_{n}} P\left(X_{n}^{l-1}=x_{j} \mid I_{n}^{l-1}=x_{i}\right)\right)
\end{aligned}
$$

It may be worthwhile to emphasize that the last term in (6) is necessary since when a fault is misclassified as another fault (but not as "healthy") in a component at level $l-1$, the health state of the child component remains the same (faulty).

Case 2: Computation of $P\left(X_{p}^{l}=x_{k} \mid X_{1, \ldots, N}^{l-1}=x\right)$ for $k=0$

The probability $P\left(X_{p}^{l}=x_{0} \mid X_{n}^{l-1}=x_{i}\right)$ is the probability that level $l$ is at a healthy state given that it's $n$-th sub-component at level $l-1$ is at a faulty state $x_{i}$. Since this is a case of disagreement between the two levels, we use the belief adjustment factor $\left(1-h_{n, i}^{p, n}\right)$ in our following computations. Furthermore, since the state $X_{p}^{l}=x_{0}$ is dependent on all the parent subcomponents (Observation 2.1), we need to take into account the probabilities that are related to all the sub-components' healthy states as follows:

$$
\begin{aligned}
& P\left(X_{p}^{l}=x_{0} \mid X_{1, \ldots, N}^{l-1}=x\right) \\
& \approx P\left(X_{p}^{l}=x_{0} \mid I_{n}^{l-1}=x_{i}\right) \prod_{j \neq n, j=1}^{N} P\left(X_{p}^{l}=x_{0} \mid I_{j}^{l-1}=x_{0}\right) \\
& =\left(1-h_{n, i}^{p, n}\right) P\left(X_{n}^{l-1}=x_{0} \mid I_{n}^{l-1}=x_{i}\right) \\
& \prod_{j \neq n, j=1}^{N} h_{j, 0}^{p, 0} P\left(X_{j}^{l-1}=x_{0} \mid I_{j}^{l-1}=x_{0}\right)
\end{aligned}
$$

Case 3: Computation of $P\left(X_{p}^{l}=x_{k} \mid X_{n}^{l-1}=x_{i}\right)$ for $k \neq 0$ and $k \neq n$

As in Case 2 above, this is a case of disagreement as well. However, in this case since $k \neq 0$ and $k \neq n$, when the level $l$ is at the state $x_{k}$ there is no dependency that is represented in our dependency model (Observation 2.1) through which $x_{k}$ can be related to the health state of the $n$-th sub-component at level $l-1$. Therefore, the set of probabilities $P\left(X_{p}^{l}=x_{k} \mid X_{n}^{l-1}=x_{i}\right)$, $k=1, \ldots, N(k \neq 0$ and $k \neq n)$ represent uncertainties that are related to un-modeled dependencies. Given that the distribution in (3) has to satisfy the constraint in (4), we propose to distribute beliefs equally among the set as follows:

$$
\begin{aligned}
& P\left(X_{p}^{l}=x_{k} \mid X_{n}^{l-1}=x_{i}\right) \\
& =\frac{1}{N-1}\left(1-P\left(X_{p}^{l}=x_{n} \mid X_{n}^{l-1}=x_{i}\right)\right. \\
& \left.\quad-P\left(X_{p}^{l}=x_{0} \mid X_{1, \ldots, N}^{l-1}=x\right)\right)
\end{aligned}
$$

where $k \neq 0$ and $k \neq n$. The procedure for computing $P\left(X_{p}^{l}=\right.$ $\left.x_{n} \mid X_{n}^{l-1}=x_{i}\right)$ and $P\left(X_{p}^{l}=x_{0} \mid X_{1, \ldots, N}^{l-1}=x\right)$ are described in Case 1 and Case 2, respectively.

Next, in order to determine the distribution in (2) we observe that $X_{p}^{l}=x_{0}$ only when all the parent sub-components are healthy. To avoid any ambiguity, we denote the distribution in (2) by $P\left(X_{p}^{l} \mid X_{1, \ldots, N}^{l-1}=x_{0}\right)$. By Assumption 2.1, we propose to compute the distribution as follows:

$$
\begin{aligned}
& P\left(X_{p}^{l}=x_{0} \mid X_{1, \ldots, N}^{l-1}=x_{0}\right) \\
& =1-P\left(X_{p}^{l}=\overline{x_{0}} \mid X_{1, \ldots, N}^{l-1}=x_{0}\right) \\
& =1-\prod_{j=1}^{N}\left(1-h_{j, 0}^{p, 0}\right) P\left(X_{j}^{l-1}=\overline{x_{0}} \mid I_{j}^{l-1}=x_{0}\right)
\end{aligned}
$$

where $\overline{x_{0}}$ corresponds to the set $\left\{X_{p}^{l}\right\} \backslash x_{0}$ or $\left\{X_{n}^{l-1}\right\} \backslash x_{0}$ depending on the level in the hierarchy. The remaining probabilities in the distribution in (2), i.e., $P\left(X_{p}^{l}=x_{k} \mid X_{1, \ldots, N}^{l-1}=x_{0}\right)$ where $k=1, \ldots, N$, represent un-modeled dependencies since according 
to Observation 2.1, non-faulty states at level $l-1$ are not mapped to faulty states at level $l$. In such a situation, as in Case 3 above, we propose to distribute beliefs equally among the set as follows:

$$
\begin{aligned}
& P\left(X_{p}^{l}=x_{k} \mid X_{1, \ldots, N}^{l-1}=x_{0}\right) \\
& =\frac{1}{N}\left(1-P\left(X_{p}^{l}=x_{0} \mid X_{1, \ldots, N}^{l-1}=x_{0}\right)\right)
\end{aligned}
$$

Computation of Initial Distributions When Nodes Are Not Actively Monitored: As mentioned in Section II-A, it may be the case that some nodes in our proposed CDM are not monitored actively. Consequently, fault diagnosis algorithms are not deployed in those nodes. However, since our proposed node health state assignments follow a systematic pattern (refer to Section II-A and Observation 2.1 ), it is easy to observe that the distributions in (1) or (3) are expected to be maximum at $X_{p}^{l}=x_{n}$ assuming that the accuracy of the diagnosis algorithm satisfies the user specification $\gamma_{\text {spaec }}$. Similarly, the distribution in (2) is expected to be maximum at $X_{p}^{l}=x_{0}$. Therefore, the initial distributions are specified such that the following conditions are satisfied:

$$
\begin{aligned}
& \operatorname{argmax}_{x_{n} \in X_{p}^{l}} P\left(X_{p}^{l} \mid X_{1, \ldots, N}^{l-1}\right) \\
& = \begin{cases}x_{n} & \text { for the distributions in (1) } \\
x_{0} & \text { for the distribution in (2) }\end{cases}
\end{aligned}
$$

In order to satisfy the above conditions, in the case of missing information first we assume a near-maximum hierarchical agreement and set $h_{n, i}^{p, n}$ (in (6) and (7)), and $h_{j, 0}^{p, 0}$ (in (7) and (9)) a value that is close to 1 . Next, we assume "ideal" probabilities by setting $P\left(X_{n}^{l-1}=x_{i} \mid I_{n}^{l-1}=x_{i}\right)=\beta_{11}$ (in (6)), $P\left(X_{n}^{l-1}=\right.$ $\left.x_{0} \mid I_{n}^{l-1}=x_{i}\right)=\beta_{01}$ and $P\left(X_{j}^{l-1}=x_{0} \mid I_{j}^{l-1}=x_{0}\right)=\beta_{00}$ (in (7)), and $P\left(X_{j}^{l-1}=\overline{x_{0}} \mid I_{j}^{l-1}=x_{0}\right)=\left(1-\beta_{00}\right)$ (in (9)); where $\beta_{11} \approx \gamma_{\text {spec }}, \beta_{01} \approx\left(1-\gamma_{\text {spec }}\right), \beta_{00} \approx \gamma_{\text {spec }}$, and $\gamma_{\text {spec }}$ is the desired (design specification) probability of the correct health state given an identification in the parent nodes if suitable diagnosis algorithms were employed.

Finally, in the case of a component $C_{p}^{l-1}$ that does not have a confusion matrix available, but has a similar component $C_{q}^{l-1}$ with the same health states (for example, the reaction wheel actuators in a three-axis active attitude control subsystem) and a common child node $C_{p}^{l}$, the confusion matrix of $C_{p}^{l-1}$ may be considered to be the same as that associated with $C_{q}^{l-1}$ in order to specify the distributions in the CPT at the child node $C_{p}^{l}$. For such a set of similar components, it is also possible to construct a common confusion matrix by including data from the components.

Computation of the Remaining Distributions: Once the initial distributions are determined, we propose to compute the remaining distributions by using a weighted-sum of the initial distributions, as in (1) and (2), as follows:

$$
P\left(X_{p}^{l} \mid X_{1, \ldots, N}^{l-1}\right)=\sum_{j=1}^{N_{\lambda}+1} w_{j} P_{j}\left(X_{p}^{l} \mid X_{1, \ldots, N}^{l-1}\right)
$$

where $P\left(X_{p}^{l} \mid X_{1, \ldots, N}^{l-1}\right)$ represents $P\left(X_{p}^{l} \mid X_{1}^{l-1}, X_{2}^{l-1}, \ldots, X_{N}^{l-1}\right)$, $P_{j}$ is an initial distribution, $w_{j} \in W$, and $W$ is an $\left(N_{\lambda}+1\right)$ dimensional weight vector that is subjected to the constraint $\sum_{j=1}^{N_{\lambda}+1} w_{j}=1$. It is suggested that the human experts are provided with the initial distributions and are asked to decide the weights $w_{j}$ based on their judgements. Therefore, given the initial distributions, our proposed procedure would require that the number of weight parameters $w_{j}$ grows linearly with the total number of the parent nodes' health states.

It is worthwhile to note that as pointed out in [32], it is easy for the human experts to express their opinions in terms of such weight assignments. Therefore, eventhough our procedure is simple, it is consistent with how human experts develop their beliefs by starting from some "anchor" values and adjusting them to specify probabilities (adjustment and anchoring heuristics) [41]. Alternatively, one may choose to develop a weight assignment policy that is based on prior probabilities of the faults in the initial distributions under consideration. However, in order to minimize biases towards certain types of faults that are frequently identified, the policy should include other considerations such as component operating hours since some faults may develop only toward the end of life of the component whereas others may develop at the early stages. Development of such a policy is beyond the scope of this work and has been left as part of our future work.

\section{Evidence Generation}

By evidence generation at a node $C_{p}^{l}$ with health states $x_{k} \in$ $X_{p}^{l}, k=0,1, \ldots, K$ we refer to the construction of a $K$ dimensional vector $\mathbf{e}_{p}^{l}=\left\{x_{0}=0, \ldots, x_{k}=1, \ldots, x_{K}=0\right\}$ of zeros and ones that is used to instantiate the node when its health state is identified as $x_{k}$ by employing a suitable fault diagnosis algorithm. As mentioned in Section II-B, component/node health states are identified by employing appropriate/available fault diagnosis algorithms in our CDM nodes. We obtain fault evidences by utilizing fuzzy Rule-Based Reasoning (RBR) at various nodes of our proposed CDM. The methodology for performing fuzzy RBR at different levels of the hierarchy was presented in our earlier work [6], [9]. Here we provide an overview of the method for sake of completeness.

To perform fuzzy RBR, with a given node/component $C_{p}^{l}$, we associate diagnostic signals, fault manifestations, and fault signature models that are denoted by $S_{p}^{l}, M_{p}^{l}$, and $F S M_{p}^{l}$, respectively. By utilizing our earlier experiences on both synthetic and actual telemetry data [22], [26], we extract features from process states and/or variables and utilize them as diagnostic signals. A fault manifestation of a specific fault in our work is considered to be some pre-identified value(s) of a diagnostic signal that indicates the presence of that fault, and the fault signature is represented in the form of rule(s) that identifies all the fault manifestations of interest for that specific fault. A fault signature model of a component refers to a set of rules that corresponds to all the faults that are to be identified in that component.

One or more rules are specified for each possible health state $x_{k} \in X_{p}^{l}$ of $C_{p}^{l}$. In the general case, for a given health state $x_{i}$ and $N_{d}$ diagnostic signals $s_{n}^{l}$, we synthesize fuzzy rule(s) in the following form:

$$
\begin{aligned}
& \text { If }\left(s_{1}^{l} \in M_{1, k}^{l}\right) \text { and }\left(s_{2}^{l} \in M_{2, k}^{l}\right), \ldots, \text { and }\left(s_{N_{d}}^{l} \in M_{N_{d}, k}^{l}\right) \\
& \text { then } x_{k}
\end{aligned}
$$

where $x_{k} \in X_{p}^{l}$, and $M_{n, k}^{l} \in M_{p}^{l}$ is a (set of) value(s) of the $n$-th diagnostic signal (characterized by the fuzzy membership function(s)) when the component health state is $x_{k}$. The health state with maximum rule activation level is considered as the identified fault in the node. It is important to note that the fuzzy rule activation values are not considered as probabilities since they are fundamentally different from each other. If $p a\left(C_{p}^{l}\right)=\emptyset$, the 
rules corresponding to the faulty states of $C_{p}^{l}$ identify faults that originate in $C_{p}^{l}$ with some common and reasonable assumptions in diagnosis, such as non-simultaneous faults within a time step over which the health state of $C_{p}^{l}$ is identified and introduced to our proposed CDM.

On the other hand, if $p a\left(C_{p}^{l}\right) \neq \emptyset$, the health state of $C_{p}^{l}$ is identified by the rules that are synthesized based on the relative behavior specifications of $p a\left(C_{p}^{l}\right)$ (sub-components of $C_{p}^{l}$ ) assuming that the interactions of the sub-components are characterized by a leader-follower or master-slave control configuration and ensuring that the master component is in its healthy state before determining the rule activation levels of the slave subcomponents. More detail and discussions on the conditions for fault identifiability are available in [6].

Once the health state $x_{k} \in X_{p}^{l}$ of $C_{p}^{l}$ is identified by employing the above procedure (or any other reasoning algorithm that is employed at $C_{p}^{l}$ ) at a given instant, an evidence over the $K$ possible states of $C_{p}^{l}$ is generated as follows: $\mathbf{e}_{p}^{l}=\left\{x_{0}=\right.$ $\left.0, \ldots, x_{k}=1, \ldots, x_{K}=0\right\}$, and is subsequently introduced to the $C_{p}^{l}$ node of our proposed CDM.

\section{Fault Diagnosis Results for Satellites FORMATION FLIGHT CASE STUdY}

In this section, we demonstrate the application of the fault diagnosis methodology that was introduced in the previous section by utilizing synthetic data. Note that utilization of synthetic formation flying system data has been necessary due to the unavailability of actual telemetry data from multi-platform space missions which are still mostly in the planning and design stages. We first provide a brief description of our data generation model before presenting the fault diagnosis results.

In a leader-follower formation flight, since the leader acts as a reference point, and the formation flying mission is subjected to a single-point failure of the leader satellite, we propose to reduce the health management workload for only the follower satellites by utilizing our proposed solution. In other words, it is assumed that the leader satellite is healthy as far as our analysis is concerned. This can be ensured if we assume that the components of the leader satellite are monitored and diagnosed frequent enough to ensure that the leader is fault free before carrying out the monitoring and diagnosis of the follower satellites.

\section{A. Formation Flying Mission and System Description}

For synthetic fault data generation, first we have implemented and simulated high fidelity attitude control subsystem (ACS) and electrical power subsystem (EPS) models of a planetary environment orbit (PEO) formation flying system with 5 identical small satellites $(150 \mathrm{~kg})$ in a leader-follower (LF) configuration as shown in Figure 3. An arrow from Sat- $j$ to Sat- $i$ indicates that attitude measurements of Sat- $i$ are available with respect to Sat- $j$. A Sun-synchronous Lower Earth Orbit (LEO) with $550 \mathrm{~km}$ altitude (orbital period: $95 \mathrm{~min}$ (approximately)) was selected as the leader's Keplerian orbit. Followers are assumed to follow fuel-optimal trajectories around the leader [42], [43]. In the subsequent paragraphs we briefly introduce and describe the two subsystems that are used for generating the synthetic data for demonstrating and illustrating our fault diagnosis approach. A detailed description of the system under consideration is available in our earlier work [9].

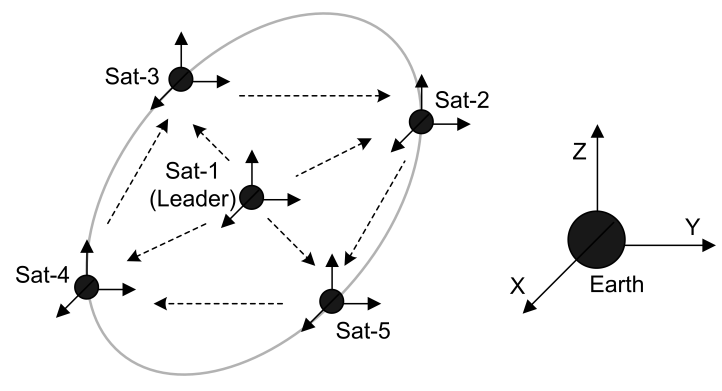

Fig. 3. Formation flight of five satellites.

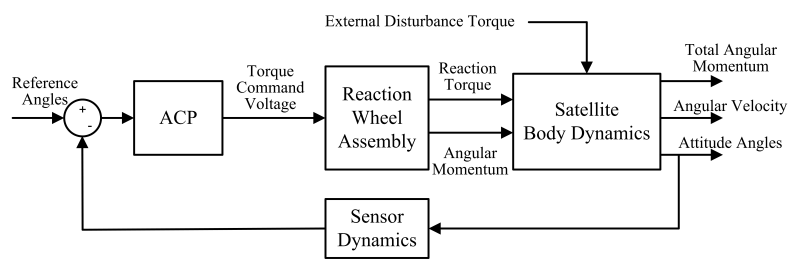

(a) Functional diagram of the ACS.

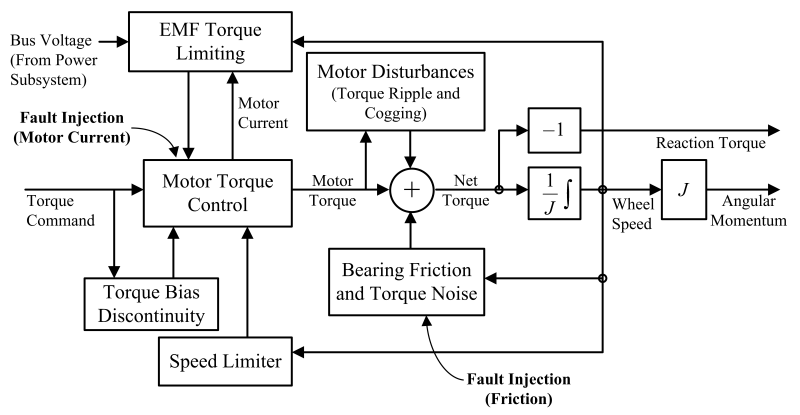

(b) A simplified schematic of a RW (adopted from [46]).

Fig. 4. Functional diagram of the ACS and a simplified schematic diagram of its reaction wheel (RW) component.

Attitude Control Subsystem: The main purpose of an attitude control subsystem (ACS) is to orientate the main structure/body of a satellite at desired angle(s) within required accuracy that is set by the payload, communication devices, etc. mounted on the main structure of the satellite. Attitude of a satellite can be represented in different ways with sets of variables such as Euler angles, direction cosine matrix (DCM), Euler parameters (also known as quaternion), etc. To avoid the well-known shortcoming of the Euler angles for large rotations, in this work we have utilized quaternion representation [44], [45] to implement the attitude dynamics of each satellite. However, for diagnosis purposes, it is convenient to work with Euler angles as they provide easy visualization of the rotations and orientation of the satellite. Hence, the ground support personnel responsible for fault diagnosis are more comfortable dealing with Euler angles. We assume a fixed formation configuration; i.e., the followers are required to maintain the same attitude as that of the leader relative to the inertial frame. The functional diagram of the implemented model of the ACS for an individual satellite is shown in Figure 4(a), in which the "ACP" consists of the PID controllers corresponding to the three axes of the satellite's body frame and the "Reaction Wheel Assembly" consists of the corresponding set of three actuators that are used for maintaining the required attitude of the "Satellite Body". For synthetic data generation, we have also incorporated a high fidelity mathematical model of the 


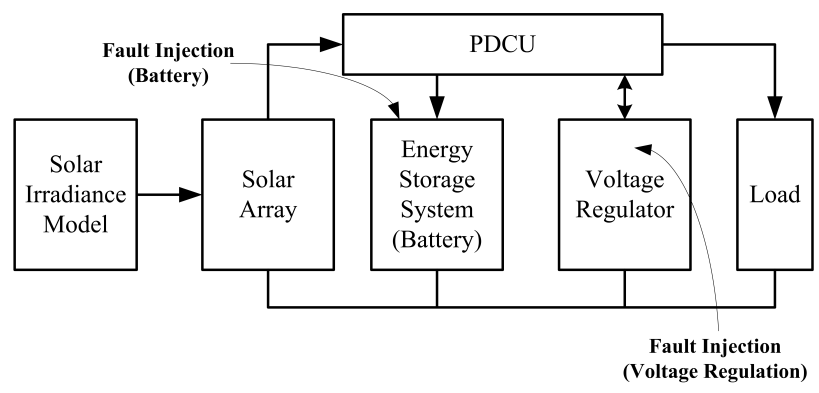

Fig. 5. Functional diagram of the electrical power subsystem (EPS) (adopted from [47], [48]) depicting the locations of fault injections under the assumed EPS fault scenarios.

Ithaco Type-A reaction wheel (RW) that is available in [46] and is shown in Figure 4(b). Worst-case environmental disturbances such as the magnetic torque, gravity-gradient torque, atmospheric drag, and solar radiation pressure corresponding to the selected orbit have been applied to the attitude dynamics model. Finally, ideal attitude sensors (operate without any transients, error or time delay) have been assumed.

Electrical Power Subsystem: We have incorporated a simplified satellite electrical power subsystem (EPS) model with our ACS model for each satellite. The EPS model [47], is modified which is available in [48] and is developed in the virtual test bed (VTB) environment [47], [49], to incorporate fault injection capabilities and to ensure the supply of the desired bus voltage to the ACS reaction wheels. A functional diagram of the EPS model of an individual satellite is shown in Figure 5, which consists of a solar array illumination model, solar array model, the controller or simplified power distribution and control unit (PDCU), a battery model, and a voltage regulator that delivers regulated bus voltage to the load; i.e., in our case, the reaction wheels.

It is important to note that the interaction between the ACS and the EPS is of the master-slave type in the sense that any fault in the EPS may manifest in the ACS but the converse is not true. Such simulation setup is justified by the fact that the target ACS fault severities do not lead to excessively large deviations in the satellite attitude that may significantly affect the Sun pointing of the solar arrays, and hence the performance of the EPS.

Fault Models (refer to Figure 6): Within the ACS, two types of intermittent faults are considered and are injected at the subsystem component (reaction wheel) level, namely $(a)$ friction fault (increase in the viscous friction), and $(b)$ reaction wheel motor current fault (decrease in the motor gain). Within the EPS, two types of faults are considered at the subsystem level, namely (a) intermittent bus voltage drop due to the voltage regulator malfunctioning, and $(b)$ intermittent bus voltage drop due to the anomaly in the battery.

Each fault is injected with 3 (three) severity levels, that is gradually increasing from the lowest to the maximum severity and then gradually decreasing before a complete fault removal. Note that the faults considered are intermittent and non-abrupt in nature. Although, we are considering component level faults within the ACS, the faults corresponding to the EPS are considered at the subsystem level due to the lack of detailed models of the EPS components within the EPS. Consequently, the EPS fault diagnosis is performed only up to the subsystem level.

\section{B. Implementation of Our Proposed Model and Fault Diagnosis Results}

We have implemented a 4-level Bayesian network-based Component Dependency Model (CDM) for the formation flight of 5 satellites that was described earlier (refer to Figure 3). Towards this end, we have used the open source $\mathrm{BN}$ tool that is available from [50]. We have used the well-known recursive conditioning algorithm [8] for belief propagation and updating. Figure 6 shows the implemented CDM where "Sat-1" ... "Sat-5" represent the five satellites in the formation, and "RW-X", "RW-Y", and "RW-Z" represent the reaction wheels (RW) in the $X, Y$, and $Z$ directions, respectively. First, we assign the states of the components $C_{p}^{l}$ with $p a\left(C_{p}^{l}\right)=\emptyset$ by following the procedure that was described in Section II. Each of the 15 RWs, denoted as $C_{i}^{1}$; where $i=1, \ldots, 15$ at level 1 (identified as the "subsystem component level") is assigned the following 3 health states: $X_{i}^{1}=$ $\{$ Healthy, friction fault, currentfault $\}$ (the fault models were discussed above). Each of the 5 electrical power subsystem (EPS) nodes, denoted as $C_{i}^{2}$; where $i=2,4,6,8,10$ at level 2 (identified as the "subsystem level") is assigned the following 3 health states: $X_{i}^{2}=\{$ Healthy, regulator fault, battery fault $\}$ (the fault models were discussed above). We assume that at the beginning of the formation operation, the system is healthy and assign prior probabilities $X_{i}^{1}=\{0.9,0.05,0.05\}$; where $i=1, \ldots, 15$, and $X_{i}^{2}=\{0.9,0.05,0.05\}$; where $i=2,4,6,8,10$, that represent the above assumption.

We assign the states of the components with $p a\left(C_{p}^{l}\right) \neq \emptyset$ by following the procedure that was specified in Section II. Each of the 5 attitude control subsystems (ACS) nodes, denoted as $C_{i}^{2}$; where $i=1,3,5,7,9$ at level 2 is assigned the following 4 health states: $X_{i}^{2}=\{$ Healthy, RW.Xfault, RW.Y fault, RW.Zfault $\}$. Each of the system level (level 3) nodes or satellites is assigned the following 3 health states: $X_{i}^{3}=\{$ Healthy, ACSfault, EPSfault $\}$. In the case of the formation component $\left(C_{1}^{4}\right)$, note that $\left|p a\left(C_{1}^{4}\right)\right|=5$ and each parent has 3 states which would lead to a large $\left(3^{5}\right)$ number of parent configurations. Consequently, first we have implemented 5 intermediate nodes between the levels 3 and 4 (not shown in Figure 6 to avoid confusions) which we denote as " $C_{1}^{4}, C_{i}^{3}$ "; where $i=1, \ldots, 5$. We assign to each of the " $C_{1}^{4}, C_{i}^{3}$ " nodes 2 states $\{$ Healthy, Sat.ifault $\}$. Finally, we assign to the formation component $\left(C_{1}^{4}\right) 3$ health states: $X_{1}^{4}=\{$ Healthy, Leader fault, follower Fault $\}$. Since the health states of the nodes " $C_{1}^{4}, C_{i}^{3}$ " and $C_{1}^{4}$ are binary, we have implemented a noisy-OR model (as mentioned in Section II) above level 3 . In the nodes with $p a\left(C_{p}^{l}\right) \neq \emptyset$, we specify CPTs by following our proposed procedure in Section II.

In the subsequent discussion, we demonstrate how the CPTs in the ACS nodes in Figure 6 are specified by using our proposed procedure. Since we are considering identical satellites, we constructed a single confusion matrix for the 15 RWs $\left(C_{1}^{1}, \ldots, C_{15}^{1}\right)$. Therefore, in this case, the CPTs that are specified in each of the 5 ACS nodes $C_{i}^{2}(i=1,3,5,7,9)$ are the same. For demonstration purposes and without loss of generality, we consider the ACS of only Sat-1, i.e., the node $C_{1}^{2}$ in the subsequent discussion.

Specification of the CPTs: To specify the CPT node $C_{1}^{2}$, first note that the parent nodes are three RWs; i.e., pa $\left(C_{1}^{2}\right)=\left\{C_{1}^{1}, C_{2}^{1}, C_{3}^{1}\right\}$. Therefore, the number of parent nodes is $N=3$. Consequently, the possible number of health states of $C_{1}^{2}$ is $N+1=4$, which are given by $X_{1}^{2}=\left\{x_{0}, x_{1}, x_{2}, x_{3}\right\}=\left\{\right.$ Healthy, $C_{1}^{1}$ fault, 


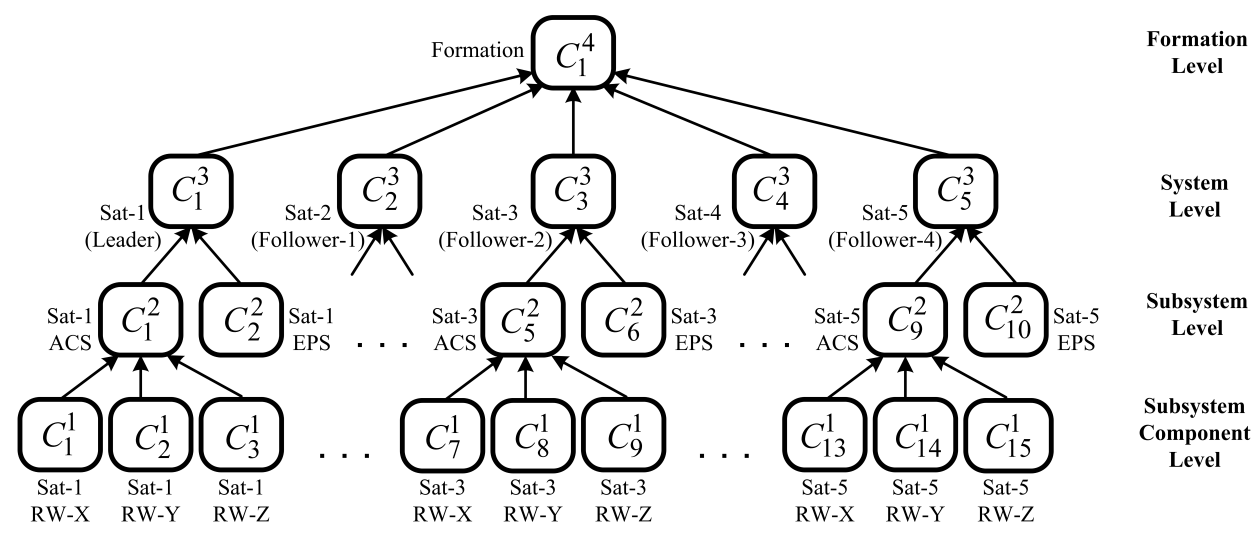

Fig. 6. A 4-level Bayesian network-based component dependency model (CDM) for hierarchical fault diagnosis.

$C_{2}^{1}$ fault, $C_{3}^{1}$ fault $\}$. The possible number of the health states of the parent nodes are $\left(m_{1}+1\right)=\left(m_{2}+1\right)=\left(m_{3}+1\right)=3$. The possible states of each of the parent nodes, as mentioned above, are $X_{i}^{1}=\left\{x_{0}, x_{1}, x_{2}\right\}=\left\{H, f_{f}, f_{c}\right\}$; where $i=1,2,3$, $H$ represents "Healthy", $f_{f}$ represents a "friction fault", and $f_{c}$ represents a "current fault". The total number of distributions that are required to be specified is $\prod_{n=1}^{N}\left(m_{n}+1\right)=27$. Now, we need to identify the $N_{\lambda}+1$ initial distributions over $X_{1}^{2}$, where $N_{\lambda}=$ $\sum_{j=1}^{N} m_{j}=6$. The $N_{\lambda}+1=7$ initial distributions over $X_{1}^{2}$ are given as follows:
(a) $P\left(X_{1}^{2} \mid X_{1}^{1}=x_{0}, X_{2}^{1}=x_{0}, X_{3}^{1}=x_{0}\right)$
(b) $P\left(X_{1}^{2} \mid X_{1}^{1}=x_{1}, X_{2}^{1}=x_{0}, X_{3}^{1}=x_{0}\right)$
(c) $P\left(X_{1}^{2} \mid X_{1}^{1}=x_{2}, X_{2}^{1}=x_{0}, X_{3}^{1}=x_{0}\right)$
(d) $P\left(X_{1}^{2} \mid X_{1}^{1}=x_{0}, X_{2}^{1}=x_{1}, X_{3}^{1}=x_{0}\right)$
(e) $P\left(X_{1}^{2} \mid X_{1}^{1}=x_{0}, X_{2}^{1}=x_{2}, X_{3}^{1}=x_{0}\right)$
(f) $P\left(X_{1}^{2} \mid X_{1}^{1}=x_{0}, X_{2}^{1}=x_{0}, X_{3}^{1}=x_{1}\right)$
(g) $P\left(X_{1}^{2} \mid X_{1}^{1}=x_{0}, X_{2}^{1}=x_{0}, X_{3}^{1}=x_{2}\right)$

Note that the initial distribution ( $a$ ) above corresponds to equation (2) and the remaining distributions correspond to equation (1). To specify the distribution $(a)$ above, we need to compute the following conditional probabilities: (a.1) $P\left(X_{1}^{2}=x_{0} \mid X_{1}^{1}=\right.$ $\left.x_{0}, X_{2}^{1}=x_{0}, X_{3}^{1}=x_{0}\right)$, (a.2) $P\left(X_{1}^{2}=x_{1} \mid X_{1}^{1}=x_{0}, X_{2}^{1}=\right.$ $\left.x_{0}, X_{3}^{1}=x_{0}\right)$, (a.3) $P\left(X_{1}^{2}=x_{2} \mid X_{1}^{1}=x_{0}, X_{2}^{1}=x_{0}, X_{3}^{1}=x_{0}\right)$, and (a.4) $P\left(X_{1}^{2}=x_{3} \mid X_{1}^{1}=x_{0}, X_{2}^{1}=x_{0}, X_{3}^{1}=x_{0}\right)$.

Note that we have employed fuzzy rule-based component fault diagnosis (refer to Section II-C) in the following nodes of Figure 6 that is the 15 RWs $\left(C_{1}^{1}, \ldots, C_{15}^{1}\right)$, the five EPSs $\left(C_{2}^{2}, C_{4}^{2}, C_{6}^{2}, C_{8}^{2}, C_{10}^{2}\right)$, and the formation component $\left(C_{1}^{4}\right)$. For computing the conditional probability $(a .1)$, we refer to equation (9). Since the confusion matrices that are associated with the three parent nodes $C_{1}^{1}, C_{2}^{1}$, and $C_{3}^{1}$ are the same, the corresponding belief adjustments factor are the same. In addition, since we do not have any diagnosis algorithm deployed in $C_{1}^{2}$, the information (the values $a_{x_{n}}^{2} ; n=0,1,2,3$; refer to the policy that is related to the belief adjustment factor as mentioned in Section II-B) necessary to determine the belief adjustment factor is not available. However, in this case, we have $a_{x_{i}}^{1} ; i=0,1,2$. We assume $a_{x_{n}}^{2}=0.95$; for $n=0,1,2,3$ (close to 1 as mentioned in Section II-B), and from the "one-versus-all" decision matrices (as described in Section II-B) that are obtained from the confusion matrices (associated with the employed rule based reasoning at nodes $C_{1}^{1}$, $C_{2}^{1}$, and $C_{3}^{1}$, and not shown here due to space limitations), we have, $a_{x_{0}}^{1}=0.937, a_{x_{1}}^{1}=0.893$, and $a_{x_{2}}^{1}=0.941$. Consequently, we have $h_{1,0}^{1,0}=h_{2,0}^{1,0}=h_{3,0}^{1,0}=0.937 / 0.95=0.986, h_{1,1}^{1,1}=$ $h_{2,1}^{1,2}=h_{3,1}^{1,3}=0.893 / 0.95=0.940$, and $h_{1,2}^{1,1}=h_{2,2}^{1,2}=$ $h_{3,2}^{1,3}=0.941 / 0.95=0.991$. Since all the parent nodes are associated with the same confusion matrix, as indicated earlier from the confusion matrix we obtain, $P\left(X_{1}^{1}=\overline{x_{0}} \mid I_{1}^{1}=x_{0}\right)=$ $P\left(X_{2}^{1}=\overline{x_{0}} \mid I_{2}^{1}=x_{0}\right)=P\left(X_{3}^{1}=\overline{x_{0}} \mid I_{3}^{1}=x_{0}\right)=0.071$. With these values, the conditional probability $(a .1)$ is computed as follows: $P\left(X_{1}^{2}=x_{0} \mid X_{1}^{1}=x_{0}, X_{2}^{1}=x_{0}, X_{3}^{1}=x_{0}\right)=$ $1-\left\{\left(1-h_{1,0}^{1,0}\right) P\left(X_{1}^{1}=\overline{x_{0}} \mid I_{1}^{1}=x_{0}\right)\left(1-h_{2,0}^{1,0}\right) P\left(X_{2}^{1}=\overline{x_{0}} \mid I_{2}^{1}=\right.\right.$ $\left.\left.x_{0}\right)\left(1-h_{3,0}^{1,0}\right) P\left(X_{3}^{1}=\overline{x_{0}} \mid I_{3}^{1}=x_{0}\right)\right\}=0.999$.

For computing the conditional probabilities (a.2), (a.3), and (a.4) we refer to equation (10) and compute the following probabilities: $P\left(X_{1}^{2}=x_{i} \mid X_{1}^{1}=x_{0}, X_{2}^{1}=x_{0}, X_{3}^{1}=x_{0}\right)=$ $(1 / N)\left(1-P\left(X_{1}^{2}=x_{0} \mid X_{1}^{1}=x_{0}, X_{2}^{1}=x_{0}, X_{3}^{1}=x_{0}\right)\right)=0.0003$. Therefore, the initial distribution $(a)$ is computed as $P\left(X_{1}^{2} \mid X_{1}^{1}=\right.$ $\left.x_{0}, X_{2}^{1}=x_{0}, X_{3}^{1}=x_{0}\right)=(0.999,0.0003,0.0003,0.0003)$. Next, to specify the distribution $(b)$, we need to compute the following probabilities: (b.1) $P\left(X_{1}^{2}=x_{0} \mid X_{1}^{1}=x_{1}, X_{2}^{1}=x_{0}, X_{3}^{1}=x_{0}\right)$, (b.2) $P\left(X_{1}^{2}=x_{1} \mid X_{1}^{1}=x_{1}, X_{2}^{1}=x_{0}, X_{3}^{1}=x_{0}\right)$, (b.3) $P\left(X_{1}^{2}=\right.$ $\left.x_{2} \mid X_{1}^{1}=x_{1}, X_{2}^{1}=x_{0}, X_{3}^{1}=x_{0}\right)$, and (b.4) $P\left(X_{1}^{2}=x_{3} \mid X_{1}^{1}=\right.$ $\left.x_{1}, X_{2}^{1}=x_{0}, X_{3}^{1}=x_{0}\right)$.

For computing the conditional probability $(b .2)$, we refer to equation (6). The value $P\left(X_{1}^{1}=x_{1} \mid I_{1}^{1}=x_{1}\right)=0.644$ is obtained from the confusion matrix. Using the value of the belief adjustment factor that was computed earlier as $h_{1,1}^{1,1}=0.940$, the conditional probability is obtained as follows: $P\left(X_{1}^{2}=x_{1} \mid X_{1}^{1}=\right.$ $\left.x_{1}\right)=h_{1,1}^{1,1}\left(P\left(X_{1}^{1}=x_{1} \mid I_{1}^{1}=x_{1}\right)+P\left(X_{1}^{1}=x_{2} \mid I_{1}^{1}=x_{1}\right)\right)$ $=0.877$.

For computing (b.1), we refer to equation (7) and use the conditional probabilities that are available from the confusion matrix according to the following computations: $P\left(X_{1}^{2}=x_{0} \mid X_{1}^{1}=\right.$ $\left.x_{1}\right)=P\left(X_{1}^{2}=x_{0} \mid I_{1}^{1}=x_{1}\right) \prod_{j=2}^{3} P\left(X_{1}^{2}=x_{0} \mid I_{j}^{1}=x_{0}\right)$ $=\left(1-h_{1,1}^{1,1}\right) P\left(X_{1}^{1}=x_{0} \mid I_{1}^{1}=x_{1}\right) h_{2,0}^{1,0} P\left(X_{2}^{1}=x_{0} \mid I_{2}^{1}=x_{0}\right)$ $h_{3,0}^{1,0} P\left(X_{3}^{1}=x_{0} \mid I_{3}^{1}=x_{0}\right)=0.003$.

For computing (b.3) and (b.4), we refer to equation (8) and compute the conditional probabilities as follows: $P\left(X_{1}^{2}=x_{k} \mid X_{1}^{1}=\right.$ $\left.x_{1}\right)=(N-1)\left(1-P\left(X_{1}^{2}=x_{1} \mid X_{1}^{1}=x_{1}\right)-P\left(X_{1}^{2}=x_{0} \mid X_{1}^{1}=\right.\right.$ $\left.\left.x_{1}\right)\right)=0.060$.

Therefore, the initial distribution $(b)$ is obtained as $P\left(X_{1}^{2} \mid X_{1}^{1}=\right.$ $\left.x_{1}, X_{2}^{1}=x_{0}, X_{3}^{1}=x_{0}\right)=(0.003,0.877,0.060,0.060)$. By following the same procedure, the initial distribution $(c)$ is computed as $P\left(X_{1}^{2} \mid X_{1}^{1}=x_{2}, X_{2}^{1}=x_{0}, X_{3}^{1}=x_{0}\right)=$ $(0.000,0.991,0.0045,0.0045)$. Now, since we have a single confusion matrix for all the three parent nodes, in this particular 
case, the remaining initial distributions, i.e., $(d),(e),(f)$, and (g) are obtained as $P\left(X_{1}^{2} \mid X_{1}^{1}=x_{0}, X_{2}^{1}=x_{1}, X_{3}^{1}=x_{0}\right)$ $=(0.003,0.060,0.877,0.060), P\left(X_{1}^{2} \mid X_{1}^{1}=x_{0}, X_{2}^{1}=x_{2}, X_{3}^{1}=\right.$ $\left.x_{0}\right)=(0.000,0.0045,0.991,0.0045), P\left(X_{1}^{2} \mid X_{1}^{1}=x_{0}, X_{2}^{1}=\right.$ $\left.x_{0}, X_{3}^{1}=x_{1}\right)=(0.003,0.060,0.060,0.877)$, and $P\left(X_{1}^{2} \mid X_{1}^{1}=\right.$ $\left.x_{0}, X_{2}^{1}=x_{0}, X_{3}^{1}=x_{2}\right)=(0.000,0.0045,0.0045,0.991)$, respectively.

The remaining distributions in the CPTs are generated by using equation (12). As an example, the distribution associated with two different faults (a "friction fault" in the RW-1 and a "current fault" in the RW-3) is computed by assigning weights (this should be assigned by the human expert) as $P\left(X_{1}^{2} \mid X_{1}^{1}=x_{1}, X_{2}^{1}=x_{0}, X_{3}^{1}=\right.$ $\left.x_{2}\right)=w_{2}(b)+w_{7}(g)=(0.0015,0.4407,0.0323,0.5255)$; where $w_{2}=0.5$ and $w_{7}=0.5$ (the two faults are believed to be eually possible), and the remaining weights are set to zero. The computation of the distribution $(g)$ and that of the other distributions are quite similar and are not shown here due to space limitations.

Node Health State Identification and Evidence Generation: In order to generate the health state evidences that are to be introduced at different nodes of our CDM, we have performed a fuzzy rule-based component fault diagnosis (refer to Section II-C) for the following components of Figure 6. Specifically, we have the 15 RWs $\left(C_{1}^{1}, \ldots, C_{15}^{1}\right)$, the five EPSs $\left(C_{2}^{2}, C_{4}^{2}, C_{6}^{2}, C_{8}^{2}, C_{10}^{2}\right)$, and the formation component $\left(C_{1}^{4}\right)$.

For the health state identification of the RWs $\left(C_{1}^{1}, \ldots, C_{15}^{1}\right)$, we have obtained the diagnostic signals by extracting features from the following reaction wheel (RW) measurements, namely (a) motor current, (b) torque command voltage, and (c) wheel speed. Based on our earlier experience with an actual attitude control subsystem telemetry data [22], we have extracted simple features that include (over an invocation window of 512 seconds; that is approximately 11 invokes per orbit) mean, standard deviation, minimum value, peak value, energy, and the first 5 components of the Fast Fourier Transform (FFT) energy spectrum. The rules corresponding to the healthy RW condition as well as to the two faults under consideration are determined (the general form of the rules is given in equation (13) and the details are available in [6] and are not discussed here), and the rule activations in each invocation are computed.

Similarly, to identify the health states of the EPSs $\left(C_{2}^{2}, C_{4}^{2}, C_{6}^{2}\right.$, $C_{8}^{2}, C_{10}^{2}$ ), we have obtained the diagnostic signals by extracting features from the following EPS measurements, namely (a) bus voltage, (b) regulator output current, and (c) battery current. We have extracted simple features that include (over the abovementioned invocation window of 512 seconds) mean, standard deviation, minimum value, and the one component of the Fast Fourier Transform (FFT) energy spectrum. Rules corresponding to the healthy EPS condition as well as to the two faults under consideration are determined by utilizing the above features. For the health state identification of the formation $\left(C_{1}^{4}\right)$, mean of the relative attitude measurements over an invocation window is utilized as a diagnostic signal. As in the case of RWs, the rule activations in each invocation are computed for EPSs and for the formation level. Note that the health state evidences corresponding to the formation node are introduced at the intermediate nodes (as indicated earlier). Here, as an example, for fault diagnosis of the formation component $\left(C_{1}^{4}\right)$, we depict the Sat-3 rule activation when the above-mentioned friction fault was injected in the $\mathrm{Z}$-axis reaction wheel (subsystem component level) between $t=7500$ sec and $t=9480$ sec. Figure 7 shows the rule activations
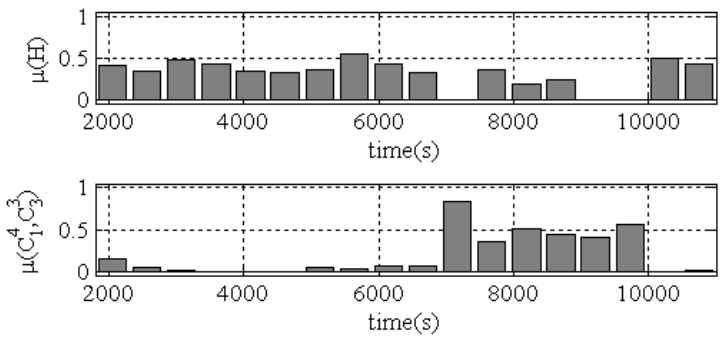

Fig. 7. Sat-3 rule activations (the width of each bar-graph is 512 seconds).

in the intermediate node " $C_{1}^{4}, C_{3}^{3}$ " (as explained earlier) where $\mu\left(C_{1}^{4}, C_{3}^{3}\right)$ and $\mu(H)$ represent the rule activations corresponding to a faulty and a healthy condition of Sat-3, respectively. Given the rule activations as shown in Figure 7 , an evidence $\mathbf{e}=\{0,1\}$ (which is introduced in the intermediate node as described above) is generated whenever the rule activation $\mu\left(C_{1}^{4}, C_{3}^{3}\right)>\mu(H)$ is satisfied.

Hierarchical Diagnosis: We have introduced fault evidences at different levels to our CDM, and have propagated evidences and updated belief distributions of the CDM nodes by using the wellknown recursive conditioning algorithm [8]. In most cases, the computed distributions clearly justified the existence of faults. For example, the fault evidence $\mathbf{e}_{9}^{1}=\{0,1,0\}$ (friction fault at Sat-3 $\mathrm{RW}-\mathrm{Z}$ ) is introduced, the probability distributions over the health states of Sat-3 under the fault free condition as well as under the injected friction fault at Sat-3 RW-Z are shown in Figure 8. The distributions clearly justify the existence of a fault in the ACS.

The distributions corresponding to the other fault scenarios are not graphically presented here due to space limitations. Instead, the accuracy of the hierarchical fault diagnosis results are summarized under various scenarios in the next section.

\section{Performance Evaluation and Validation}

In the subsequent paragraphs, we evaluate the performance, identify advantages and limitations, and describe possible validation methods of our proposed method.

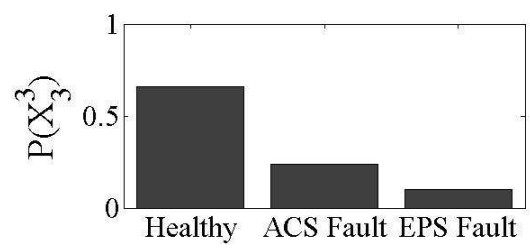

(a) Before the presence of the fault.

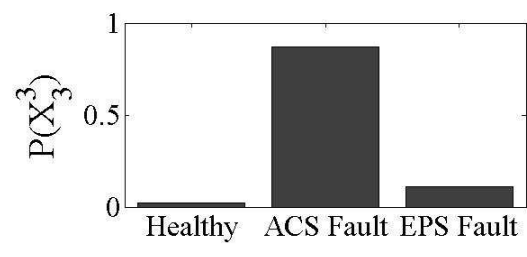

(b) During the presence of the fault.

Fig. 8. $\quad \mathrm{P}\left(X_{3}^{3}\right)$ of Sat-3 when an evidence of a fault is introduced at the subsystem component level. 
Model Accuracy: We evaluate the performance of our proposed $\overline{\mathrm{CDM}}$ by using a set of fault scenarios under which the actual health states of all the nodes in the CDM are known. The accuracy is computed in terms of the \% of nodes in the CDM that are "representative" of their true known health states. The representativeness is determined as follows: let a fault evidence $\mathbf{e}_{p_{1}}^{l_{1}}$ be introduced at node $C_{p_{1}}^{l_{1}}$ and the known actual health state of a component $C_{p_{2}}^{l_{2}}$ be $x^{*} \in X_{p_{2}}^{l_{2}}$. Furthermore, let $\pi\left(C_{p_{1}}^{l_{1}}\right)$ and $\sigma\left(C_{p_{1}}^{l_{1}}\right)$ be the sets of predecessors and successors of node $C_{p_{1}}^{l_{1}}$, respectively in the CDM. We consider the health state of $C_{p_{2}}^{l_{2}}$ to be representative of its true health state $x^{*}$ when the following specifications or conditions hold:

1) Let $C_{p_{2}}^{l_{2}} \in \sigma\left(C_{p_{1}}^{l_{1}}\right)$, then belief about $X_{p_{2}}^{l_{2}}=x^{*}$ increases with the introduction of the evidence; i.e., $P\left(X_{p_{2}}^{l_{2}}=\right.$ $\left.x^{*} \mid \mathbf{e}_{p_{1}}^{l_{1}}\right)>P\left(X_{p_{2}}^{l_{2}}=x^{*} \mid \emptyset\right)$.

2) Let $C_{p_{2}}^{l_{2}} \in \pi\left(C_{p_{1}}^{l_{1}}\right)$ and the actual known state be faulty $\left(x^{*} \neq x_{0}\right)$, then belief about $X_{p_{2}}^{l_{2}}=x^{*}$ increases with the introduction of the evidence, and the belief is higher than (or, at least equal to) that of other fault states at level $l_{2}$; i.e., $P\left(X_{p_{2}}^{l_{2}}=x^{*} \mid \mathbf{e}_{p_{1}}^{l_{1}}\right)>P\left(X_{p_{2}}^{l_{2}}=x^{*} \mid \emptyset\right)$ and $P\left(X_{p_{2}}^{l_{2}}=\right.$ $\left.x^{*} \mid \mathbf{e}_{p_{1}}^{l_{1}}\right) \geq P\left(X_{p}^{l_{2}}=x_{j} \mid \mathbf{e}_{p_{1}}^{l_{1}}\right)$; where $x_{j} \neq x_{0}, x_{j} \neq x^{*}$ and $p$ represents the component at level $l_{2}$ at which no evidence has been introduced.

3) Let $C_{p_{2}}^{l_{2}} \in \pi\left(C_{p_{1}}^{l_{1}}\right)$ and the actual known state be healthy $\left(x^{*}=x_{0}\right)$, then the belief about the faults in $C_{p_{2}}^{l_{2}}$ are lower than that in the faulty components at level $l_{2}$; i.e., $P\left(X_{p_{2}}^{l_{2}}=\right.$ $\left.x \mid \mathbf{e}_{p_{1}}^{l_{1}}\right)<P\left(X_{p}^{l_{2}}=x_{j} \mid \mathbf{e}_{p_{1}}^{l_{1}}\right)$; where $x \in\left\{X_{p_{2}}^{l_{2}} \backslash x^{*}\right\}, x_{j} \neq x_{0}$ and $p$ represents the faulty component, if any, at level $l_{2}$ at which no evidence has been introduced.

If there is no known faulty component at level $l_{2}$ (fault originated at $C_{p_{3}}^{l_{3}}, l_{1}<l_{3}<l_{2}$ ), then the belief distribution over $X_{p_{2}}^{l_{2}}$ is maximum at $x^{*}$ and $P\left(X_{p_{2}}^{l_{2}}=x \mid \mathbf{e}_{p_{1}}^{l_{1}}\right)<$ $P\left(X_{p_{3}}^{l_{3}}=x_{j} \mid \mathbf{e}_{p_{1}}^{l_{1}}\right)$; where $x \in\left\{X_{p_{2}}^{l_{2}} \backslash x^{*}\right\}$ and $x_{j} \neq x_{0}$.

4) Let $C_{p_{2}}^{l_{2}} \notin\left\{\pi\left(C_{p_{1}}^{l_{1}} \cup \sigma\left(C_{p_{1}}^{l_{1}}\right)\right\}\right.$, then the belief distribution over $X_{p_{2}}^{l_{2}}$ remains unchanged with the introduction of the evidence; i.e., $P\left(X_{p_{2}}^{l_{2}} \mid \mathbf{e}_{p_{1}}^{l_{1}}\right)=P\left(X_{p_{2}}^{l_{2}} \mid \emptyset\right)$.

Table I shows the computed accuracy under various scenarios with single as well as multiple fault evidences that are introduced in the follower satellites in the CDM as shown in Figure 6. By "conflicting" evidence in Table I we imply that for the two evidences that are available in two nodes connected through a directed path, one evidence indicates a healthy state while the other indicates a faulty state. Such a scenario is possible when, for example, a fault evidence in a node at the high level is obtained but at the low level the fault is not identified or vice-versa. Note that although the scenarios are not the only possible cases, they represent some of the most common cases that may occur in practice. The average accuracy is computed over all the scenarios under consideration. Row 1 represents scenarios where a single fault evidence is introduced at a given node and the accuracy is computed for each scenario (by using the conditions above). It is observed that when one additional evidence is introduced (refer to row 2), the average accuracy increases. However, if the additional evidence is conflicting and the mis-identification is at the low level, the average accuracy decreases (refer to row 3). Furthermore, if the additional evidence is conflicting but the mis-identification is at the high level, the accuracies remain unchanged (refer to row 4). Finally, the last row shows accuracies for two simultaneous faults in two different follower satellites where the scenarios consist of both conflicting and non-conflicting evidences.

As in any large Bayesian network model, building a BNbased hierarchical fault diagnosis model as proposed in this paper involves a careful trade-off between a rich hand-crafted model versus generic dependency model. The design considerations to take into account are model parameters and result accuracies, the cost of construction (including the demand for human experts' time), maintenance (including the cost of model updating), and the complexity of the probabilistic inference. Consequently, in practice, building such a model requires multiple iterations over these tasks until a satisfactory model and solution is achieved. Below, we provide a brief discussion on some important aspects of our proposed methodology.

Advantages and Comparisons: First it should be noted that our proposed component dependency model (CDM) is a generic model that can be used to decompose a complex system hierarchically in order to perform coherent fault diagnosis. The model is generic in the sense that $(a)$ the health states of a given component is not defined subjectively, rather they are identified by the known dominant fault modes of the component, and (b) our proposed method does not impose any restrictions on the type of diagnosis algorithms that one may employ at a given node of the model as long as its performance evaluation matrix is available. Furthermore, our proposed method for obtaining model parameters overcomes the limitations of the other probability elicitation methods [30]-[32] as discussed in Section II-B.

Second, our proposed approach requires less demand on domain experts' time for obtaining the model parameters, which is known to be a costly commodity. Instead of entirely depending on interviewing domain experts, the initial distributions are obtained from node fault diagnosis performance data and known health state dependencies. This minimizes the well-known limitations of eliciting probabilities exhaustively with domain experts.

Third, our model parameters are easy to update when node performance matrix changes due to the availability of new data and improved versions of the node fault diagnosis algorithm. In this case, the initial distributions can be re-computed by following our proposed well-defined procedure (this avoids the repetition of the time consuming interview of domain experts), and the weights, if necessary, may be updated.

Finally, for formation flight fault diagnosis, our proposed CDM enables one to hierarchically decompose a complex system in order to use the data that are available from different system components systematically, and to perform diagnostic reasoning coherently. By propagating fault evidences from a node in the $\mathrm{CDM}$, one is able to update the probable health state of the other nodes, and perform in-depth investigation of the nodes of interest only (based on the updated health states). This avoids exhaustive plotting and trend analysis across a large number of components manually which requires extensive effort by human operators. Therefore, our approach has the potential for reducing the size of the operations team. Furthermore, since the performance evaluation matrix data represents an expert human's observations to a great extent, and the method obtains model parameters by utilizing such matrices, less-experienced personnel will be benefited while performing monitoring and diagnosis tasks at ground stations by utilizing our proposed model.

Note that there will always be a trade-off between a rich/detailed hand-crafted model versus a generic dependency 
TABLE I

PERformance EVAluation OF THE IMPLEMENTED CDM

\begin{tabular}{|c||c|c|c|c|c|}
\hline $\begin{array}{c}\text { Number of } \\
\text { Evidences }\end{array}$ & $\begin{array}{c}\text { Number of } \\
\text { Scenarios }\end{array}$ & $\begin{array}{c}\text { Maximum Accuracy } \\
\text { (in \% Nodes) }\end{array}$ & $\begin{array}{c}\text { Minimum Accuracy } \\
\text { (in \% Nodes) }\end{array}$ & $\begin{array}{c}\text { Average Accuracy } \\
\text { (in \% Nodes) }\end{array}$ & Comments \\
\hline \hline \multicolumn{7}{|c|}{ Single Fault in a Follower Satellite } \\
\hline 1 & 16 & $100 \%$ & $83.33 \%$ & $97.91 \%$ & None \\
\hline 2 & 20 & $100 \%$ & $100 \%$ & $100 \%$ & $\begin{array}{c}\text { Evidences introduced } \\
\text { at different levels }\end{array}$ \\
\hline 2 & 8 & $91.67 \%$ & $86.11 \%$ & $90.28 \%$ & $\begin{array}{c}\text { Conflicting evidence } \\
\text { at levels } 1 \text { or } 2\end{array}$ \\
\hline 2 & 8 & $100 \%$ & $100 \%$ & $100 \%$ & $\begin{array}{c}\text { Conflicting evidence } \\
\text { at formation level }\end{array}$ \\
\hline \hline \multicolumn{7}{|c|}{ Two Simultaneous Faults in Two Follower Satellites } \\
\hline $2,3,4$ & 72 & $100 \%$ & $77.78 \%$ & $90.43 \%$ & $\begin{array}{c}\text { Different evidence types } \\
\text { and combinations }\end{array}$ \\
\hline
\end{tabular}

model. It is clear from the above discussion that our proposed model will reduce the cost of model construction (the demand for human experts' time) and maintenance (the cost of model updating).

The framework of the proposed hierarchical fault diagnosis approach was first introduced briefly in our earlier work in [5]. However, that work did not discuss component dependency models. Later on a hierarchical fault diagnosis approach similar to the current work was developed in [6], where it was possible to specify the dependencies only with absolute certainty by using ones and zeros to indicate connectivity/dependency as opposed to using BN-based models and CPTs. Consequently, if a fault evidence is found at a given node, fuzzy rule activations had to be computed at all the parent nodes, and it was not possible to propagate beliefs to update and estimate health states without computing the rule activations. Given the fault evidence(s) and updated belief distributions, in our present work we compute the rule activations at only those nodes that have high fault probabilities to confirm the identification of a fault. The work in [6] does not propose any Bayesian network-based model for hierarchical fault diagnosis. Our work in [9] presents the idea of our proposed CDM but does not provide any detail on the computations of the model parameters. Our work in [10] presents the computations of CPTs which are explained in detail in the current paper along with the detailed explanation of our proposed approach, a clear and revised presentation of the proposed framework, and a detailed description of the formation flight system under consideration. Finally, performance evaluation results associated with our proposed CDM have been included in the current paper, and a possible validation method was also discussed.

Limitations: The main limitation of our proposed method is that the faults that originate in a component at a particular level are implicitly assumed to be non-interfering with the diagnostic signals of other components (that have a common child node) at the same level. This assumption is reasonable when the fault diagnosis algorithms that are deployed in those nodes are designed to identify faults with a severity range that is low enough not to affect the performances of the other nodes in the same level. Consequently, their influences on the child nodes are to be considered as independent. Note that this limitation arises from the type of information that is made available to our model development. Specifically, according to our problem in hand, the node fault diagnosis algorithms are developed in isolation, and are often proprietary to the development teams. Nevertheless, one should investigate the validity of the independence assumptions by using design information and experimental data as reported in our earlier work [9].

Validation Methods: One way to validate our proposed model would be to compare the initial distributions (and hence the CPTs) that are specified by using our proposed procedure with those that are obtained by using the expert beliefs or another method, if available. As an alternative, we have been investigating well-known formal Verification and Validation (V\&V) techniques for Bayesian networks such as sensitivity analysis [37], [51] to validate our proposed model. Biases that are introduced by the prior probabilities and the inaccuracies in conditional probabilities influence the reliability of a BN model's output. Sensitivity analysis is a technique for systematic investigation of the influences of the model input and parameters on its outputs (which may result due to the changes in accuracies in a node diagnosis and leading to a change in the corresponding confusion matrix). Since a brute-force method which involves the variation of every single conditional probability, for performing sensitivity analysis is both highly time-consuming and computationally an intensive process, our ongoing research is focused on the development of a formal validation procedure for our proposed model. However, this problem is beyond the scope of this paper and is not investigated any further.

\section{CONCLUSiOnS AND Future WORK}

In this paper, we have developed a hierarchical fault diagnosis methodology which allows systematic and coherent fault diagnosis in different components or subsystems of a complex formation flight of satellites. The general idea is to decompose a complex system hierarchically into simpler modules or nodes, and perform diagnostic reasoning hierarchically by utilizing the fault diagnosis algorithms that are deployed at different nodes and which are connected via our proposed Bayesian network-based Component Dependency Model (CDM). The model structure was developed from the knowledge of the component health state dependencies. A methodology for determining model parameters was developed which demands considerably less effort from the domain experts, and easy to update when node fault diagnosis 
performances change. To determine the probability distributions that are required and that need to be specified in the Conditional Probability Tables (CPT), our proposed method obtains certain initial probability distributions from the node fault diagnosis performance matrices. Subsequently, by taking the domain experts' opinion into account, the remaining probability distributions are specified.

We have demonstrated the effectiveness of our proposed methodology by using synthetic data of a leader-follower formation flight of satellites. The data generation model consists of two subsystems, namely the attitude control subsystem and the electrical power subsystem for each satellite in the formation. We have implemented our proposed CDM by decomposing the formation flying system hierarchically into 4 levels. We have introduced the evidences that are generated via fuzzy rule-based reasoning of faults at different levels in the hierarchy. Our results show that when fault evidences are introduced at a node, the states of the remaining nodes of the implemented CDM are updated to reflect the correct health states of the corresponding components.

As part of our ongoing as well as future work, we plan to investigate the validation of our proposed model as well as to conduct cost-benefit analysis in a practical environment with real system data. Extension of the proposed CDM to a dynamic Bayesian network-based CDM will also be an interesting research problem that may be pursued.

\section{REFERENCES}

[1] J. Kurien and M. D. R-Moreno, "Cost and Benefits of Model-based Diagnosis," in Proc. 2008 IEEE Aerospace Conference, Big Sky, Montana, USA, March 2008, pp. 1-14.

[2] D. L. Iverson, "System Health Monitoring for Space Mission Operations," in Proc. 2008 IEEE Aerospace Conference, Big Sky, Montana, USA, March 2008, pp. 1-8.

[3] J. Korbicz, J. M. Koscieny, Z. Kowalczuk, and W. Cholewa, Eds., Fault Diagnosis: Models, Artificial Intelligence, Applications. Germany: Springer, 2004.

[4] R. Isermann, "Model-based Fault Detection and Diagnosis-Status and Applications," Annual Reviews in Control, vol. 29, pp. 71-85, 2005.

[5] A. Barua and K. Khorasani, "Intelligent Model-based Hierarchical Fault Diagnosis for Satellite Formations," in Proc. 2007 IEEE International Conference on Systems, Man and Cybernetics (SMC 2007), Montreal, Canada, October 2007.

[6] —_, "A Hierarchical Fault Diagnosis and Fuzzy Rule-Based Reasoning for Satellites Formation Flight," Accepted for publication in IEEE Transactions on Aerospace and Electronic Systems, 2010.

[7] J. Pearl, Probabilistic Reasoning in Intelligent Systems: Networks of Plausible Inference. San Monteo, CA, USA: Morgan Kaufmann, 1988.

[8] F. V. Jensen and T. D. Nielsen, Bayesian Networks and Decision Graphs, 2nd ed. NY, USA: Springer, 2007.

[9] A. Barua and K. Khorasani, "Hierarchical Fault Diagnosis and Health Monitoring in Multi-platform Space Systems," in Proc. 2009 IEEE Aerospace Conference, Big Sky, Montana, USA, March 2009.

[10] — - "Hierarchical Fault Diagnosis in Satellites Formation Flight," in Proc. Annual Conference of the PHM Society, San Diego, California, USA, September-October 2009.

[11] R. Gessner, B. Kosters, A. Hefler, R. Eilenberger, J. Hartmann, and M. Schmidt, "Hierarchical FDIR Concepts in S/C Systems," in Proc. 8th International Conference on Space Operations (SpaceOps2004), Montreal, Canada, May 2004.

[12] A. Mohammadi-Idghamishi and S. Hashtrudi-Zad, "Hierarchical Fault Diagnosis: Application to an Ozone Plant," IEEE Transactions on Systems, Man, and Cybernetics - Part C: Applications and Reviews, vol. 37, No. 5, pp. 1040-1047, September 2007.

[13] M. Blanke, M. Kinnaert, L. Lunze, and M. Staroswiecki, Diagnosis and Fault-Tolerant Control, 2nd ed. Germany: Springer, 2006.

[14] R. R. Yager, "On the Construction of Hierarchical Fuzzy Systems Models," IEEE Transactions on Systems, Man, and Cybernetics - Part C: Applications and Reviews, vol. 28, No. 1, pp. 55-66, February 1998.
[15] W. Wang, "An Enhanced Diagnostic System for Gear System Monitoring," IEEE Transactions on Systems, Man, and Cybernetics - PART B: Cybernetics, vol. 38, No. 1, pp. 102-112, February 2008.

[16] Y. Zheng, H. Fang, and H. O. Wang, "TakagiSugeno Fuzzy-ModelBased Fault Detection for Networked Control Systems with Markov Delays," IEEE Transactions on Systems, Man, and Cybernetics - PART B: Cybernetics, vol. 36, No. 4, pp. 924-929, August 2006.

[17] R. Ganguli, "Application of Fuzzy Logic for Fault Isolation of Jet Engines," Journal of Engineering for Gas Turbines and Power, vol. 125, No. 3, pp. 617-623, July 2003.

[18] W. Wang, "An Intelligent System for Machinery Condition Monitoring," IEEE Transactions on Fuzzy Systems, vol. 16, No. 1, pp. 110-122, February 2008.

[19] T. Yairi, Y. Kato, and K. Hori, "Fault Detection by Mining Association Rules from House-keeping Data," in Proc. International Symposium on Artificial Intelligence, Robotics and Automation in Space, 2001.

[20] R. Mackey, M. James, H. Park, and M. Zak, "BEAM: Technology for Autonomous Self-Analysis," in IEEE Aerospace Conference, Big Sky, MT, March 2001, pp. 2989-3001.

[21] H. A. Talebi, K. Khorasani, and S. Tafazoli, "A Recurrent Neural Network-Based Sensor and Actuator Fault Detection and Isolation for Nonlinear Systems with Application to the Satellite's Attitude Control Subsystem," IEEE Transactions on Neural Networks, vol. 20, No. 1, pp. 45-60, January 2009.

[22] A. Joshi, V. Gavriloiu, A. Barua, A. Garabedian, P. Sinha, and K. Khorasani, "Intelligent and Learning-based Approaches for Health Monitoring and Fault Diagnosis of RADARSAT-1 Attitude Control System," in Proc. 2007 IEEE International Conference on Systems, Man and Cybernetics (SMC 2007), Montreal, Canada, October 2007.

[23] I. A. Al-Zyoud and K. Khorasani, "Neural Network-based Actuator Fault Diagnosis for Attitude Control Subsystem of an Unmanned Space Vehicle," in Proc. IEEE International Joint Conference on Neural Networks (IJCNN-2006), Vancouver, Canada, July 2006.

[24] Z. Q. Li, L. Ma, and K. Khorasani, "A Dynamic Neural Network-based Reaction Wheel Fault Diagnosis for Satellites," in Proc. IEEE International Joint Conference on Neural Networks (IJCNN-2006), Vancouver, Canada, July 2006.

[25] A. Valdes and K. Khorasani, "A Pulsed Plasma Thruster Fault Detection and Isolation Strategy for Formation Flying of Satellites," Applied Soft Computing, vol. doi:10.1016/j.asoc.2009.09.005, September 2009.

[26] A. Barua, P. Sinha, and K. Khorasani, "A Diagnostic Tree Approach for Fault Cause Identification in the Attitude Control Subsystem of Satellites," IEEE Transactions on Aerospace and Electronic Systems, vol. 45, No. 3, pp. 983-1002, July 2009.

[27] N. Tudoroiu and K. Khorasani, "Satellite Fault Diagnosis using a Bank of Interactive Kalman Filters," IEEE Transactions on Aerospace and Electronic Systems, vol. 43, No. 4, pp. 1334-1350, October 2007.

[28] S. N. Huang, K. K. Tan, and T. H. Lee, "Automated Fault Detection and Diagnosis in Mechanical Systems," IEEE Transactions on Systems, Man, and Cybernetics - Part C: Applications and Reviews, vol. 37, No. 6, pp. 1360-1364, November 2007.

[29] X. Dai, Z. Gao, T. Breikin, and H. Wang, "Disturbance Attenuation in Fault Detection of Gas Turbine Engines: A Discrete Robust Observer Design," IEEE Transactions on Systems, Man, and Cybernetics - Part C: Applications and Reviews, vol. 39, No. 2, pp. 234-239, March 2009.

[30] S. Renooij, "Probability Elicitation for Belief Networks: Issues to Consider," The Knowledge Engineering Review, vol. 163, No. 3, pp. 255-269, 2000.

[31] D. Nikovski, "Constructing Bayesian Networks for Medical Diagnosis from Incomplete and Partially Correct Statistics," IEEE Transactions on Knowledge and Data Engineering, vol. 12, No. 4, pp. 509-516, July/August 2000.

[32] N. E. Fenton, M. Neil, and J. G. Caballero, "Using Ranked Nodes to Model Qualitative Judgments in Bayesian Networks," IEEE Transactions on Knowledge and Data Engineering, vol. 19, No. 10, pp. 1420-1432, October 2007.

[33] D. Bernard, G. Doraist, E. Gamble, B. Kanefskyt, J. Kurien, G. K. Man, W. Millart, N. Muscettola, P. Nayak, K. Rajant, N. Rouquette, B. Smith, W. Taylor, and Y. Tung, "Spacecraft Autonomy Flight Experience: The DS1 Remote Agent Experiment," in Proc. AIAA, Albuquerque, NM, 1999.

[34] B. C. Williams and P. P. Nayak, "A Model-based Approach to Reactive Self-Configuring Systems," in Proc. AAAI-96, Portland, Oregon, August 1996, pp. 971-978.

[35] M. L. James and L. P. Dubon, "An Autonomous Diagnostic and Prognostic Monitoring System for NASA's Deep Space Network," in 
Proc. IEEE Aerospace Conference, Big Sky, MT, March 2000, pp. 403414.

[36] "Health and Usage Monitoring Metrics, Monitoring the Monitor," Society of Automotive Engineers (SAE) Standard, Issuing Committee: E32 Aerospace Propulsion Systems Health Management, February 2008, Document No. ARP 5783.

[37] M. Bednarskia, W. Cholewa, and W. Frid, "Identification of Sensitivities in Bayesian Networks," Engineering Applications of Artificial Intelligence, vol. 17, pp. 327-335, 2004.

[38] T. Kurtoglu, O. J. Mengshoel, and S. Poll, "A Framework for Systematic Benchmarking of Monitoring and Diagnostic System," in Proc. 2008 International Conference on Prognostics and Health Management, Denver, Colorado, USA, October 2008.

[39] T. Kurtoglu, D. Jensen, and S. Poll, "Systematic Benchmarking of Diagnostic Technologies for an Electrical Power System," in Proc. 2009 IEEE Aerospace Conference, Big Sky, Montana, USA, March 2009.

[40] K. B. Laskey and S. M. Mahoney, "Network Engineering for Agile Belief Network Models," IEEE Transactions on Knowledge and Data Engineering, vol. 12, No. 4, pp. 487-498, July/August 2000.

[41] D. Kahneman, P. Slovic, and A. Tversky, "Judgment Under Uncertainty: Heuristics and Biases," in Judgment Under Uncertainty: Heuristics and Biases, D. Kahneman, P. Slovic, and A. Tversky, Eds. Cambridge University Press, Cambridge, 1982, pp. 3-20.

[42] G. W. Hill, "Researches in Luner Theory," American Journal of Mathematics, vol. 1, No. 1, pp. 5-26, 1878.

[43] S. R. Vadali, S. S. Vaddi, and K. T. Alfriend, "An Intelligent Control Concept for Formation Flying Satellites," International Journal of Robust and Nonlinear Control, vol. 12, pp. 97-115, 2002.

[44] P. C. Hughes, Spacecraft Attitude Dynamics. USA: John Wiley \& Sons Inc., 1986.

[45] W. Ren and R. W. Beard, "Decentralized Scheme for Spacecraft Formation Flying via the Virtual Structure Approach," AIAA Journal of Guidance, Control and Dynamics, vol. 27, No. 1, pp. 73-82, January 2004.

[46] B. Bialke, "High Fidelity Mathematical Modeling of Reaction Wheel Performance," Advances in the Astronautical Sciences, vol. 98, pp. 483496, 1998.

[47] Z. Jiang, S. Liu, and R. A. Dougal, "Design and Testing of Spacecraft Power Systems Using VTB," IEEE Transactions on Aerospace and Electronic Systems, vol. 39, No. 3, pp. 976-989, July 2003.

[48] "Satellite Electrical Power System," USC Virtual Test Bed Homepage, Cited: July 2009. [Online]. Available: http://vtb.ee.sc.edu/applications/

[49] Z. Jiang, R. A. Dougal, and S. Liu, "Application of VTB in Design and Testing of Satellite Electrical Power Systems," Journal of Power Sources, vol. 122, pp. 95-108, 2003.

[50] “SAMIAM," Automated Reasoning Group, UCLA, Cited: July 2009. [Online]. Available: http://reasoning.cs.ucla.edu/samiam/

[51] H. Wang, "Using Sensitivity Analysis to Validate Bayesian Networks for Airplane Subsystem Diagnosis," in Proc. 2006 IEEE Aerospace Conference, Big Sky, MT, USA, March 2006.

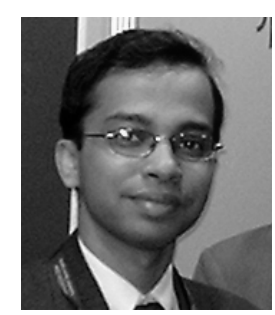

Amitabh Barua received the B. Tech. (Hons.) degree in electrical engineering from the Indian Institute of Technology (IIT) at Kharagpur, India in 2000, and the M.A.Sc. degree in electrical engineering from Concordia University, Montreal, Canada in 2004 where he is currently pursuing research toward the Ph.D. degree in electrical and computer engineering. For the last six years, he has been involved in various industrial research, technology development, and systems engineering projects/contracts that are related to control \& diagnostics of satellites and commercial aircraft subsystems. His research work is primarily focused on diagnostics, prognostics and health management (DPHM) of aerospace systems and components.

Mr. Barua is a student member of the American Institute of Aeronautics and Astronautics (AIAA).

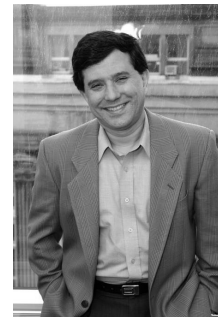

K. Khorasani (M 85) received the B.S., M.S., and Ph.D. degrees in Electrical and Computer Engineering from the University of Illinois at UrbanaChampaign in 1981, 1982 and 1985, respectively. From 1985 to 1988 he was an Assistant Professor at the University of Michigan at Dearborn and since 1988, he has been at Concordia University, Montreal, Canada, where he is currently a Professor and Concordia University Tier I Research Chair in the Department of Electrical and Computer Engineering. His main areas of research are in nonlinear and adaptive control, intelligent and autonomous control of networked unmanned systems, fault diagnosis, isolation and recovery (FDIR), neural network applications to pattern recognition, robotics and control, adaptive structure neural networks, and modeling and control of flexible link/joint manipulators. He has authored/co-authored over 250 publications in these areas. 